\title{
Assessment of Open Magnetic Fusion for Space Propulsion
}

\author{
F. Romanelli ${ }^{\mathrm{a}}$, C. Bruno ${ }^{\mathrm{b}}$ and G. Regnoli ${ }^{\mathrm{a}}$ \\ Final report of the ARIADNA study 04/3102 \\ ESTEC Contract 18853/05/NL/MV \\ ${ }^{a}$ Associazione EURATOM-ENEA sulla Fusione \\ C.R. Frascati, 00044, Frascati (Roma) Italy \\ 'DMA, Università di Roma "La Sapienza" \\ Via Eudossiana 18, 00184 Roma
}

\begin{abstract}
.
The exploration of the solar system requires advanced propulsion techniques capable of specific impulse above $10^{4} \mathrm{~s}$ and specific power in the range $1-10 \mathrm{~kW} / \mathrm{kg}$. Fusion is the most interesting option to meet these requirements. Generic fusion propulsion studies, briefly reviewed in the report, show that specific power values in the desired range are indeed feasible provided a high fraction of the fusion power can be used for direct thrust. Open magnetic field configurations are particularly suited to such purpose. Their present status, open issues and proposals for space propulsion systems based on them are reviewed. The analysis is focused on mirrors (tandem mirror and gas-dynamic mirror), field reversed configurations, spheromaks and levitated dipole. Possible topics for further studies are proposed.
\end{abstract}


Table of contents

1. Introduction 2. General issues in the use of fusion for space propulsion

2.1 Application of fusion to space propulsion

2.2 Achievement of self-sustained condition

2.3 Design of a generic fusion propulsion system

2.4 Mass budget

2.4.1 Radiator

2.4.2 Magnet

2.4.3 Cryoplant

2.4.4 Blanket

2.4.5 Conversion

2.4.6 Auxiliary systems

2.5 Specific power

2.6 Fusion power density

2.7 Summary

3. Use of open magnetic field configurations for space propulsion

3.1 Classification and present status of OMF configurations

3.2 Mirror configurations

3.2.1 The simple mirror configuration

3.2.2 The tandem mirror

3.2.3 The field reversed mirror

3.2.4 The gas dynamic trap

3.2.5 Other mirror concepts

3.2.6 Existing mirror experiments

3.2.7 Mirror studies for space propulsion

3.3 Field reversed configurations

3.3.1 Formation and equilibrium

3.3.2 Open issues

3.3.3 Existing FRC experiments

3.3.4 FRC studies for space propulsion

3.4 Spheromaks

3.4.1 Spheromak formation

3.4.2 Stability limits

3.4.3 Confinement

3.4.4Present experiments

3.5 Levitated dipole

3.6 Conclusions

4. Proposal for further studies on fusion for space application 


\section{Introduction}

The exploration of the solar system and beyond requires the development of adequate propulsion techniques. A rough but simple estimate can help in understanding the terms of the problem. To accelerate a mass $\mathrm{M}_{\mathrm{w}}$ up to a velocity $\mathrm{v}_{\mathrm{c}}$ in a time $\mathrm{T}$ requires an average thrust power $\mathrm{P}$ given by

$$
\mathrm{P}=\left(\mathrm{M}_{\mathrm{w}} \mathrm{v}_{\mathrm{c}}^{2} / 2\right) / \mathrm{T}
$$

This condition define a characteristic velocity $\mathrm{v}_{\mathrm{c}}$ given by

$$
\mathrm{v}_{\mathrm{c}} \equiv(2 \alpha \mathrm{T})^{1 / 2}
$$

with $\alpha \equiv P / M_{w}$ the so-called specific power (the thrust power per unit mass) defined here in relation with the mass of the propulsion system.

The flight distance $\mathrm{L}$ is approximately given by $\mathrm{L}=\mathrm{k}_{\mathrm{o}} \mathrm{v}_{\mathrm{c}} \mathrm{T}$, with $\mathrm{k}_{\mathrm{o}}$ being a constant of order unity which depends on the details of the flight orbit. Upon combining the previous conditions and taking $\mathrm{k}_{\mathrm{o}}=1 / 3$, it follows that the specific power is related to $\mathrm{L}$ and $\mathrm{T}$ by the following condition:

$$
\alpha(\mathrm{kW} / \mathrm{kg}) \approx 10^{-3} \mathrm{~L}(\mathrm{AU})^{2} / \mathrm{T}(\mathrm{yr})^{3}
$$

with $\mathrm{L}$ in astronomical units $\left(1 \mathrm{AU} \approx 150 \times 10^{6} \mathrm{~km}\right)$ and $\mathrm{T}$ in years.

Thus, once the mission target is determined (L) the request of a reasonable flight duration (T) sets a limit on the specific power. As an example, a mission to Mars ( $\mathrm{L} \approx 1 \mathrm{AU})$ over one month requires a specific power in the range $\alpha \approx 1.7 \mathrm{~kW} / \mathrm{kg}$. A mission to the Oort cloud ( $\mathrm{L} \approx 10^{4} \mathrm{AU}$ ) lasting $20 \mathrm{yr}$ requires a specific power in the range $\alpha \approx 12 \mathrm{~kW} / \mathrm{kg}$. Thus, values of the specific power in the range $1-10 \mathrm{~kW} / \mathrm{kg}$ are adequate for the exploration of the solar system. Note that a mission to Proxima Centauri ( $\left.\approx \approx 2.5 \times 10^{5} \mathrm{AU}\right)$, lasting less than $10 \mathrm{yr}$, would require (neglecting relativistic corrections) a specific power in excess of $6 \times 10^{4} \mathrm{~kW} / \mathrm{kg}$.

In assessing the propulsion system performance, the other figure of merit, besides the specific power, is the payload fraction. Following Stuhlinger (1964), the payload fraction can be easily determined in terms of the characteristic velocity $\mathrm{v}_{\mathrm{c}}$ defined in Eq.(1.2) from the Tsiolkovski's equation. Upon defining $M_{o} \equiv M_{L}+M_{w}+M_{p}$, with $M_{o}, M_{L}, M_{w}$, and $\mathrm{M}_{\mathrm{p}}$ being the initial, payload, propulsion system and fuel mass, respectively, and expressing $\mathrm{M}_{\mathrm{w}}$ in terms of the specific power $\left(\mathrm{M}_{\mathrm{w}}=\mathrm{P} / \alpha\right)$ it is possible to show that

$$
\mathrm{M}_{\mathrm{L}} / \mathrm{M}_{\mathrm{o}}=\exp \left(-\mathrm{v}_{\mathrm{f}} / \mathrm{v}_{\mathrm{ex}}\right)-\left(\mathrm{v}_{\mathrm{ex}} / \mathrm{v}_{\mathrm{c}}\right)^{2}\left[1-\exp \left(-\mathrm{v}_{\mathrm{f}} / \mathrm{v}_{\mathrm{ex}}\right)\right]
$$

With $\mathrm{v}_{\mathrm{f}}$ being the final velocity and $\mathrm{v}_{\mathrm{ex}}$ being the velocity of the ejected fuel (related to the specific impulse $\mathrm{I}_{\mathrm{sp}}=\mathrm{V}_{\mathrm{ex}} / \mathrm{g}$ ). Equation (1.4) shows that a positive payload fraction can 
be obtained only for $\mathrm{v}_{\mathrm{f}} / \mathrm{v}_{\mathrm{c}}=\mathrm{v}_{\mathrm{f}} /(2 \alpha \mathrm{T})^{1 / 2} \leq 0.8$ and within a finite domain of $\mathrm{v}_{\mathrm{ex}} / \mathrm{v}_{\mathrm{c}}$ (with the domain increasing as $\mathrm{v}_{\mathrm{f}} / \mathrm{v}_{\mathrm{c}}$ decreases). The optimal payload fraction is approximately obtained for $\mathrm{v}_{\mathrm{c}} \approx 2^{1 / 2} \mathrm{v}_{\mathrm{ex}}$ (see Fig. 1.1)

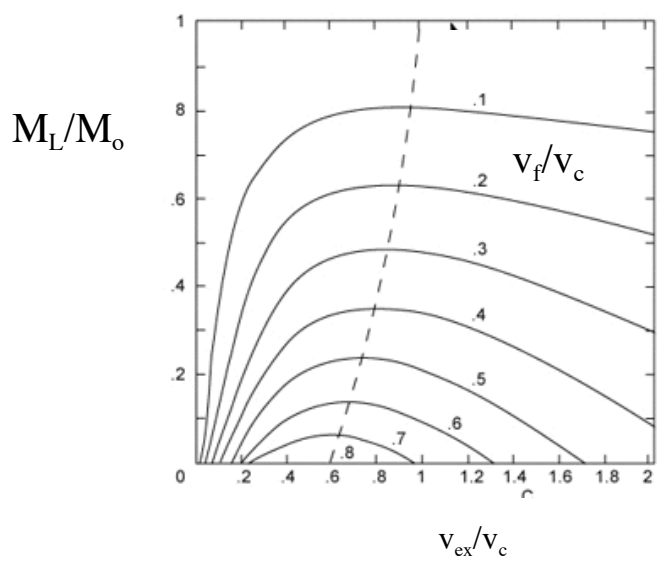

Fig.1.1 Payload fraction

It is apparent from Fig.1.1 that in order to have reasonable performance high values of specific impulse are mandatory. As an example, taking the optimal payload condition $\mathrm{v}_{\mathrm{c}}=2^{1 / 2} \mathrm{v}_{\mathrm{ex}}$, in order to fly over $1 \mathrm{AU}$ in one months with a specific power of $3.5 \mathrm{~kW} / \mathrm{kg}$ and a payload fraction $\approx 0.1\left(\mathrm{v}_{\mathrm{f}} / \mathrm{v}_{\mathrm{c}} \approx 0.7\right)$, requires a specific impulse of the order $10^{4} \mathrm{~s}$ which is well beyond the capabilities of chemical propulsion systems.

Note that the above conditions determine also the thrust per unit mass $(\mathrm{F} / \mathrm{M})$ (i.e. the average acceleration). Since $\mathrm{P} \approx \mathrm{Fv}_{\mathrm{ex}}$, it follows that

$$
\mathrm{F} / \mathrm{M} \approx 10 \mathrm{~g} \alpha(\mathrm{kW} / \mathrm{kg}) / \mathrm{I}_{\mathrm{sp}}(\mathrm{s})
$$

Such a value is larger than the gravity acceleration in the Sun field at the earth radius $\left(\approx 6 \times 10^{-4} \mathrm{~g}\right)$ for values of the specific power larger than $6 \mathrm{~kW} / \mathrm{kg}$ and $\mathrm{I}_{\mathrm{sp}}=10^{5} \mathrm{~s}$, so high thrust missions are possible for such a parameter range.

In order to achieve high specific impulse, fusion propulsion has been proposed. Indeed:

- Fusion reactions produce low-mass (atomic number $A=1-4$ ), high-energy (up to $14 \mathrm{MeV}$ ) fusion products with associated specific impulse in the range $I_{\mathrm{sp}}=4 \times 10^{6} \mathrm{~s}$.

- The reacting mixture is typically composed by $\mathrm{H}$ or $\mathrm{He}$ isotopes with average energy between $10 \mathrm{keV}$ and $100 \mathrm{keV}$. If part of such a mixture is used for propulsion, rather than the reaction products, specific impulse values in the range $5 \times 10^{4} \mathrm{~s}-2 \times 10^{5} \mathrm{~s}$ can be produced.

- Even the low-temperature plasma flowing in the region surrounding the reacting core (the so-called scrape-off region) can have temperatures in the range of $100 \mathrm{eV}$ corresponding to specific impulse values around $5 \times 10^{3} \mathrm{~s}$. 
Fusion can be applied to space propulsion in two basic ways:

- fusion electric propulsion: the fusion power is transformed in electric power either through a conventional thermodynamic cycle (in this case the wasted power must be radiated in space) or through direct conversion; the main disadvantage of this scheme is the presence of a radiator, and of all the items needed for the electricity conversion (turbines, etc.), the large mass of the electric propulsion system and the overall conversion efficiency (thermal power into thrust power);

- direct propulsion: the escaping un-reacted fuel and fusion products are expanded in a magnetic nozzle, possibly mixed with cold propellant to achieve a unidirectional jet with an optimal specific impulse. Note however that some electricity production is needed for control and auxiliary heating. In addition the ejection of un-reacted fuel requires bringing in space substantial propellant mass and must be taken into account in evaluating the overall performance.

Since fusion has the capability of producing high- $\mathrm{I}_{\mathrm{sp}}$ propellant, the possibility of its application for space propulsion depends on the possibility of building systems with specific power in the range $1-10 \mathrm{~kW} / \mathrm{kg}$ (Schulze (1994)). The aim of this report is to assess the potential capability of open magnetic field configurations to achieve such a target value. In Appendix A an example of trajectories for a Mars mission is presented showing the enabling potential of fusion propulsion, especially as far as transit time.

The application of steady-state fusion reactors to space propulsion was investigated by NASA between 1958 and 1978 (see Schulze (1991)). The research addressed the application of fusion to generate electrical power in space, as well as propulsion. These two applications are somewhat orthogonal, though the underlying plasma and fusion science are similar. The NASA Lewis program was focused on the simple mirrors and the electric field bumpy torus - both steady-state magnetic fusion energy approaches. The program was cancelled in 1978 for budgetary reasons as NASA was preparing to embark on the shuttle program. During the ' $80 \mathrm{~s}$ attention focused on the possibility of electric power generation in space over extended period of time ( $>1$ day) at the multi megawatt level. These studies (see Roth (1989) for a review) found low values for the specific power. The studies carried out since the late ' 80 s have therefore tried to optimize the fusion performance in order to maximize the specific power. Several concepts have been considered: the high-field tokamak (Bussard 1990), the spherical torus (Borowski 1995, Williams 1998), mirror systems (Kulcinski 1987, Santarius 1988, Carpenter 1992 and 1993, Kammash 1995b), field reversed configuration (Chapman 1989, Cheung 2004) and magnetic dipole (Teller 1992). These configurations will be reviewed in the context of the discussion of the different confinement systems. They are summarized in Table 1.1, which also shows the values of the specific power, the thrust power and, when available, the mass of the various components.

This report is organized as follows. In Section 2 the general issues for the use of magnetic confinement fusion for space propulsion are discussed. In Section 3 the present status of research on open magnetic field configuration is reviewed. Section 4 is devoted to a list of the possible R\&D activities for the specific application of fusion to space propulsion. 


\begin{tabular}{|l|l|l|l|l|l|l|l|l|l|l|}
\hline Reference & Configuration & $\begin{array}{c}\alpha \\
(\mathrm{kW} / \mathrm{kg})\end{array}$ & $\begin{array}{c}\text { Thrust } \\
\text { Power } \\
(\mathrm{MW})\end{array}$ & $\begin{array}{c}\text { Payload } \\
(\mathrm{t})\end{array}$ & $\begin{array}{c}\text { Struct. } \\
/ \text { Tank } \\
(\mathrm{t})\end{array}$ & $\begin{array}{c}\text { Fuel } \\
(\mathrm{t})\end{array}$ & $\begin{array}{c}\text { Rad. } \\
(\mathrm{t})\end{array}$ & $\begin{array}{c}\text { Turb. } \\
(\mathrm{t})\end{array}$ & $\begin{array}{l}\text { React. } \\
(\mathrm{t})\end{array}$ & $\begin{array}{c}\mathrm{I}_{\mathrm{sp}} \\
(\mathrm{s})\end{array}$ \\
\hline Borowski (1995) & Spheromak & 10.5 & & & & & & & & \\
\hline Borowski (1995) & $\begin{array}{l}\text { Spherical } \\
\text { Torus }\end{array}$ & 5.8 & & & & & & & & \\
\hline Santarius (1988) & $\begin{array}{l}\text { Tandem } \\
\text { Mirror }\end{array}$ & 2 & 1000 & & & & & 25 & 420 & \\
\hline Bussard (1990) & Tokamak & 3.7 & 3925 & 1900 & $\begin{array}{l}220 \\
/ 570\end{array}$ & 6310 & 760 & 170 & 70 & $5-7 \times 10^{3}$ \\
\hline Teller (1991) & Dipole & 1.0 & 1250 & & & & & & 1180 & $10^{4}-$ \\
\hline Nakashima(1994) & FRC & 1.0 & & & & & & & & $10^{3}-10^{5}$ \\
\hline Williams (1998) & $\begin{array}{l}\text { Spherical } \\
\text { Torus }\end{array}$ & 5.4 & 6145 & 108 & $/ 131$ & $45+$ & 236 & 145 & 624 & $4 \times 10^{4}$ \\
\hline Thio (1999) & MTF & 400 & 25000 & & & & 17 & & 41 & $7.7 \times 10^{4}$ \\
\hline $\begin{array}{l}\text { Kammash } \\
(1995 b)\end{array}$ & $\begin{array}{l}\text { Gasdynamic } \\
\text { trap }\end{array}$ & 7.5 & 55000 & & & & 7128 & & 100 & $1.1 \times 10^{5}$ \\
\hline Cheung (2004) & $\begin{array}{l}\text { Colliding } \\
\text { beam FRC }\end{array}$ & 3 & 100 & & 1.4 & & 6 & $18+$ & 5.5 & $1.4 \times 10^{6}$ \\
\hline Santarius (1998) & Generic DT & 0.6 & 600 & & & & 642 & & 357 & \\
\hline Santarius (1998) & GenericD- ${ }^{3} \mathrm{He}$ & 5.3 & 600 & & & & 48 & & 63.6 & \\
\hline Santarius (1998) & GenericD- ${ }^{3} \mathrm{He}$ & 10.1 & 600 & & & & 44 & & 15.8 & \\
\hline
\end{tabular}

Table 1.1 Fusion space propulsion systems studies. In the calculation of the specific power the payload and the fuel are not included. The reactor includes the auxiliary power, the batteries, the refrigerator and the magnetic nozzle. 


\section{General issues for the use of fusion in space}

In this section we first review the kinetics of the most important fusion reactions and the conditions for achieving energy amplification; in the last part a simple model for a fusion rocket is considered and a parametric expression for the specific power $\alpha$ is derived and discussed

2.1 Application of fusion for space propulsion

The starting point is the choice of fuel fusion cycles (Miley 1987). Kinetics of candidate fuels is in Table 2.1 (see also Cox 1990).

\begin{tabular}{|c|c|c|c|c|}
\hline Reaction & & Fusion fi & icles, $\mathrm{MeV}$ & $\begin{array}{c}\text { Ignition } \\
\text { temperature, }{ }^{\circ} \mathrm{C}\end{array}$ \\
\hline $1 \mathrm{a}$ & $\mathrm{D}+\mathrm{D}$ & $50 \%$ & $\mathrm{~T}(1.01)+p(3.02)$ & $300 \times 10^{6}$ \\
\hline $1 b$ & & $50 \%$ & $\mathrm{He}^{3}(0.82)+n(2.45)$ & \\
\hline 2 & $\mathrm{D}+\mathrm{T}$ & & $\mathrm{He}^{4}(3.5)+\pi(14.1)$ & $50 \times 10^{6}$ \\
\hline 3 & $\mathrm{D}+\mathrm{He}^{3}$ & 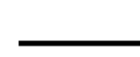 & $\mathrm{He}^{4}(3.6)+p(14.7)$ & $500 \times 10^{6}$ \\
\hline 4 & $\mathrm{~T}+\mathrm{T}$ & 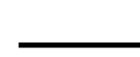 & $\mathrm{He}^{4}+2 n+11.3$ & \\
\hline Sa & $\mathrm{He}^{3}+\mathrm{T}$ & $51 \%$ & $\mathrm{He}^{4}+p+n+12.1$ & \\
\hline $5 b$ & & $43 \%$ & $\mathrm{He}^{4}(4.8)+\mathrm{D}(9.5)$ & \\
\hline $5 c$ & & $6 \%$ & $\mathrm{He}^{3}(2.4)+p(11.9)$ & \\
\hline 6 & $p+\mathrm{Li}^{6}$ & & $\mathrm{He}^{4}(1.7)+\mathrm{He}^{3}(2.3)$ & \\
\hline $7 \mathrm{a}$ & $p+\mathrm{Li}^{7}$ & $-20 \%$ & $2 \mathrm{He}^{4}+17.3$ & \\
\hline $7 b$ & & $-80 \%$ & $\mathrm{Be}^{3}+n-1.6$ & \\
\hline 8 & $\mathrm{D}+\mathrm{Li}^{6}$ & . & $3 \mathrm{He}^{4}+22.4$ & \\
\hline 9 & $p+\mathrm{B}^{11}$ & 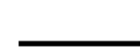 & $3 \mathrm{He}^{4}+8.7$ & \\
\hline 10 & $n+\mathrm{Li}^{6}$ & - & $\mathrm{T}+\mathrm{He}^{4}+4.8$ & \\
\hline 11 & $n+\mathrm{Li}^{7}$ & 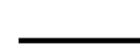 & $\mathrm{T}+\mathrm{He}^{4}-2.5$ & \\
\hline
\end{tabular}

Table 2.1 Fusion reactions

The DT reaction has the largest reactivity and can be used at relatively low temperatures $(\approx 20 \mathrm{keV})$. However, it has two main associated problems:

- $\quad 80 \%$ of the energy is produced as energetic $(14 \mathrm{MeV})$ neutrons which require a heavy shielding and the intermediate production of heat (and therefore a radiator);

- to avoid (for safety reasons) large tritium inventories, tritium must be produced in space through the conventional DT fuel cycle.

The DD reaction involves a very common isotope (33mg of Deuterium for each liter of water) and produces $33 \%$ of energy in the form of $2.45 \mathrm{MeV}$ neutrons. $14 \mathrm{MeV}$ neutrons are produced in the secondary reactions involving $\mathrm{D}$ and $\mathrm{T}$. Although the neutron problem is somewhat alleviated, energies of the reactants in the range of $100 \mathrm{keV}$ must be achieved. 
The $\mathrm{D}^{3} \mathrm{He}$ reaction needs reactant energies in the same range of the DD reaction but has the advantage of producing a very limited amount of neutrons $(\leq 15 \%)$ through the DD and the secondary DT reactions. Furthermore, the charged reaction products can be used for direct electricity conversion. Its main problem is the lack of ${ }^{3} \mathrm{He}$ on the Earth. It is envisaged to produce ${ }^{3} \mathrm{He}$ by lunar mining (estimated reserve in the range of $10^{6} \mathrm{t}$ ), see e.g. Kulcinski (2000), with a cost in the range of $\$ 400-\$ 1000 / g$. For a recent survey of the abundance of noble gases on the moon, see Ozima (2005). In perspective, it is considered in most of the studies the most promising fuel for space propulsion.

The $\mathrm{p}-{ }^{6} \mathrm{Li}$ and $\mathrm{p}-{ }^{11} \mathrm{~B}$ reactions have very low neutron production $(\approx 5 \%$ and $\approx 1 \%$, respectively) and are conventionally defined "aneutronic" (although the only truly aneutronic reaction is the ${ }^{3} \mathrm{He}-{ }^{3} \mathrm{He}$ reaction). Their main problem is the very stringent requirements to achieve a positive fusion gain. Indeed in a system with equal electron and ion temperature the amount of fusion power never exceeds the power lost via Bremsstrahlung. Thus, even in the idealized case of no losses from heat conduction, the system cannot achieve a positive fusion gain except far from thermal equilibrium (different electron and ion temperatures).

Finally, it should be mentioned that the possibility of fusion reactions catalyzed by matter-antimatter reaction has been considered for fusion propulsion systems based in inertial confinement.

\subsection{Achievement of self sustained conditions}

To achieve a significant amount of fusion reactions, the reactants must be heated at high temperature $(10-100 \mathrm{keV})$ in order to overcome the Coulomb repulsion between the positively charged nuclei. At these temperatures the electrons are no longer attached to atoms, and the state of matter is called 'plasma'. Since a plasma is composed by free charged particles it can be confined by intense magnetic fields.

The conditions to be achieved in order to obtain a significant fusion power have been discussed by Lawson (1957) and are briefly reviewed in the following. The fusion gain

$$
\mathrm{Q} \equiv \mathrm{P}_{\text {fus }} / \mathrm{P}_{\text {aux }} \text {, }
$$

defined as the ratio between the fusion power $\mathrm{P}_{\text {fus }}$ and the auxiliary power $\mathrm{P}_{\text {aux }}$ needed to heat the plasma, depends on the amount of energy losses through radiation (in the following we will consider only Bremsstrahlung) and thermal conduction. The latter are usually quantified in terms of the so-called energy confinement time $\tau_{\mathrm{E}}$ defined, in steady state conditions, as the ratio between the energy content of the plasma and the heating power. Self-sustained conditions $(\mathrm{Q}=\infty)$ are achieved when the fusion power released in the form of charged particles confined by the magnetic field balance the energy losses of the configuration and no auxiliary power is necessary. Driven operation are instead associated with a finite value of the auxiliary power and therefore of the fusion gain $\mathrm{Q}$.

The equilibrium between the plasma heating and energy losses determines the operating point of the reacting plasma and can be written as follows 


$$
\left.(1 / 2) \sum_{\mathrm{ij}} \mathrm{n}_{\mathrm{i}} \mathrm{n}_{\mathrm{j}}<\sigma v\right\rangle_{\mathrm{ij}} \mathrm{E}_{\mathrm{fus}, \mathrm{ij}}\left(\mathrm{f}_{\mathrm{ij}}+1 / \mathrm{Q}\right)=\mathrm{n}_{\mathrm{e}}{ }^{2} \mathrm{Z}_{\mathrm{eff}} \mathrm{k}_{\mathrm{Br}} \mathrm{T}_{\mathrm{e}}^{1 / 2}+(3 / 2) \Sigma_{\mathrm{i}} \mathrm{n}_{\mathrm{i}} \mathrm{T}_{\mathrm{i}} / \tau
$$

The electron density $n_{e}$ is determined by the charge neutrality condition

$$
\mathrm{n}_{\mathrm{e}}=\Sigma_{\mathrm{j}} \mathrm{n}_{\mathrm{j}} \mathrm{Z}_{\mathrm{j}}
$$

In the above expressions $n_{j}$ and $Z_{j}$ are the reacting ion species density and charge, $\langle\sigma v\rangle_{i j}$ is the reactivity (to be evaluated with the actual distribution function of the ions), $\mathrm{E}_{\text {fus,ij }}$ is the energy released in the reaction, $\mathrm{f}_{\mathrm{ij}}$ is the fraction of the fusion energy transferred to the plasma, $Z_{\text {eff }} \equiv \Sigma_{j} n_{j} Z_{j}^{2} / n_{e}$ is the effective charge, $T_{j}$ is the temperature of the $\mathrm{j}$-th species, $\mathrm{k}_{\mathrm{Br}}=1.69 \times 10^{-24} \mathrm{MWeV}^{-1 / 2}$ and $\tau$ is the energy confinement time. The above conditions define the value of $n_{e} \tau$ as a function of temperature associated with a given fusion gain $Q$. In general, optimal values of the concentrations $n_{j} / n_{e}$ can be found that minimize the $n_{e} \tau$ value. Note that the values of the fraction $\mathrm{f}_{\mathrm{ij}}$ depends both on the fraction of fusion energy released in the form of charged particles and on the capability of the configuration to confine them in the region where fusion reaction occur.

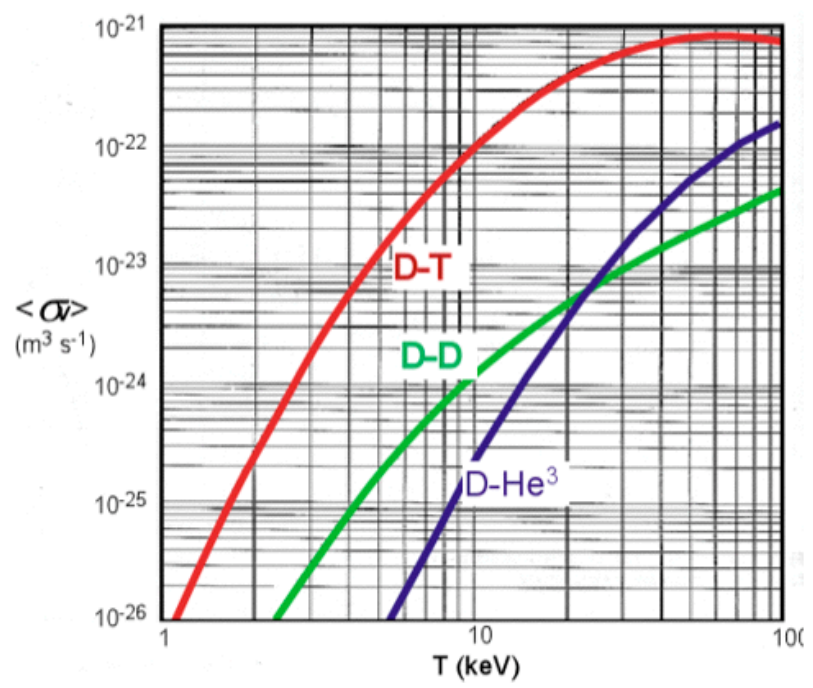

Figure 2.1 Fusion Maxwellian reactivity

Equations (2.2) determine the $n_{\mathrm{e}} \tau$ value only for the case of thermal equilibrium between all the species. In such a case all the species (electron and ions) relax to a Maxwellian distribution function with the same temperature $\mathrm{T}$ for all the species. The reactivity for this case is given by the usual Maxwellian reactivity shown in Fig.2.1. The $n_{\mathrm{e}} \tau$ value as a function of the temperature is shown in Fig.2.2. The curves show a vertical asymptote for $\mathrm{T}=\mathrm{T}_{\text {ideal }}$, the ideal ignition temperature below which the fusion power is lower than the power lost by Bremsstrahlung. A broad minimum of $n_{\mathrm{e}} \tau$ is achieved around an optimal value of the temperature $\mathrm{T}_{\mathrm{opt}}$. For much larger values of $\mathrm{T}$, the reactivity decreases and larger values of $n_{e} \tau$ are needed. The case of fully thermalized plasma $\left(T_{e}=T_{i}\right)$ allows self sustained operation only for the DT, $\mathrm{D}^{3} \mathrm{He}$ and DD reactions. In addition to the pure DD 
cycle, it is often considered the so-called "catalyzed DD" cycle, in which a small amount of $\mathrm{T}$ is added to the $\mathrm{D}$ fuel and then recovered through the DD cycle.

In some confinement scheme (especially in conjunction with the use of non conventional fuels, see e.g. Rostoker (1993)) the condition of thermal equilibrium does not apply and Eq.(2.2a) should be replaced by separate equations for the power balance of each species. In these schemes, the electrons act only as a "cold" neutralizer and Bremsstrahlung is reduced down to a level that allows a positive gain. Auxiliary power is usually supplemented in the form of energetic ion beams and the beam-beam and beam-target reactions must be accounted for. The following points must be underlined:

- The electron temperature cannot be arbitrarily small since the energetic particles (injected by the external methods or produced by fusion reactions) would be slowed down on a very fast scale by collisions with electrons.

- The fusion reactivity must be evaluated with the appropriate distribution functions for the reacting species (typically a slowing-down distribution function for the externally injected beams).

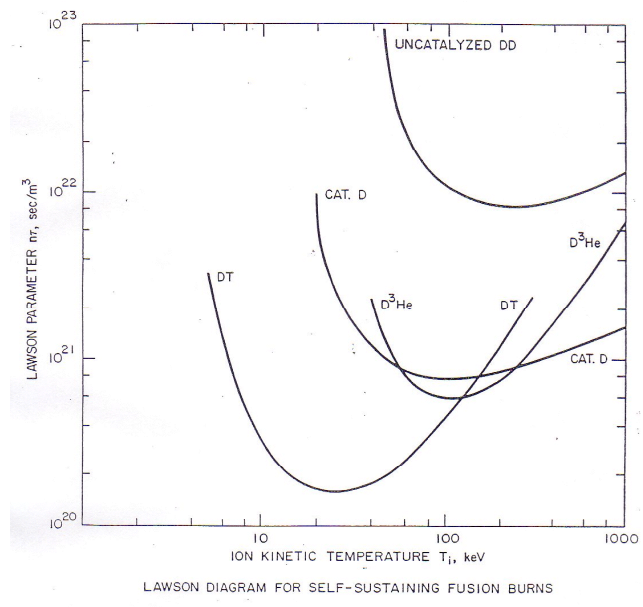

Fig. 2.2 Lawson criterion

2.3 Design of a generic fusion propulsion system

After having summarized the condition for achieving a fusion gain, we now want to discuss the trade-off between the positive and negative aspects of the various fusion systems in determining the optimal value of the specific power $\alpha$.

Following the discussion made in Santarius (1998), it is useful to determine the 


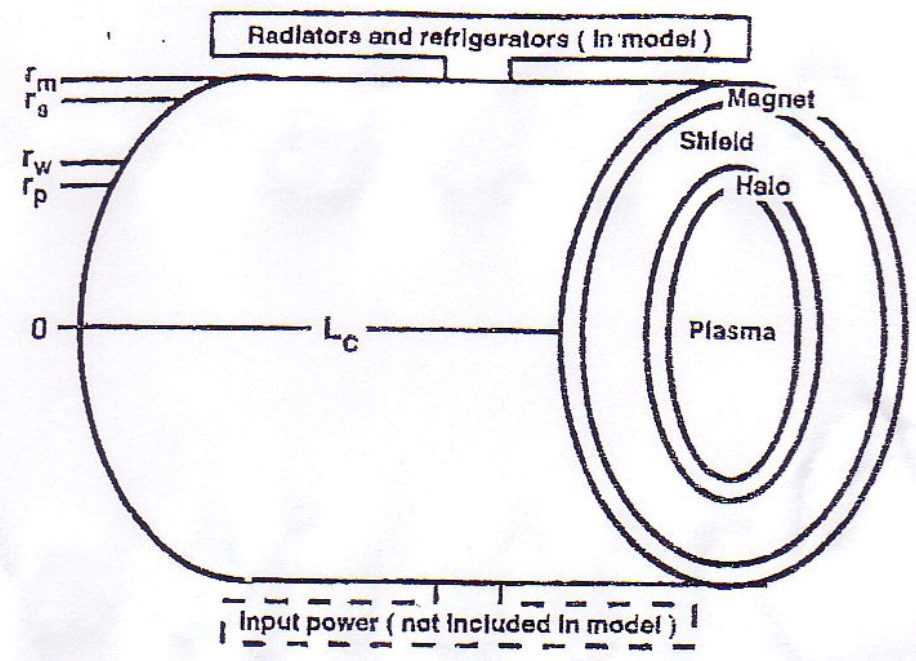

Fig.2.3 Generic Fusion Rocket geometry (from Santarius (1998))

requirements for a generic fusion propulsion system based on magnetic confinement without making reference to any specific magnetic confinement concept. In the following the system will be assumed equivalent to a cylindrical solenoid of radius $r_{m}$ and volume $V$ generating a magnetic field B (see Fig.2.3). The plasma is assumed to have a radius $r_{p}$; $a$ scrape off layer of width much lower than $r_{p}$ separates the plasma from the first wall ( adius $\mathrm{r}_{\mathrm{w}} \approx \mathrm{r}_{\mathrm{p}}$ ); the magnet is shielded by a blanket of radius $\mathrm{r}_{\mathrm{s}} \approx \mathrm{r}_{\mathrm{m}}$.

The assumed (idealized) power flow shown in Fig.2.4. The power that flows outside the reaction chamber is the sum of the fusion power plus the auxiliary power. A fraction $f_{T}$ is used directly for thrust (the case of direct propulsion or fusion electric propulsion can be modeled with a coefficient $\mathrm{f}_{\mathrm{T}}=1$ or $\mathrm{f}_{\mathrm{T}}=0$ respectively). The remaining fraction is converted either by direct conversion (for a fraction $f_{D}$ with efficiency $\eta_{D}$ ) or by thermal conversion (for the remaining part) with an efficiency $\eta_{\text {th }}$ into electrical power $\mathrm{P}_{\mathrm{el}}=\left[\eta_{\mathrm{D}} \mathrm{f}_{\mathrm{D}}+\eta_{\mathrm{th}}\left(1-\mathrm{f}_{\mathrm{D}}\right)\right]\left(1-\mathrm{f}_{\mathrm{T}}\right)\left(\mathrm{P}_{\text {fus }}+\mathrm{P}_{\text {aux }}\right)$.

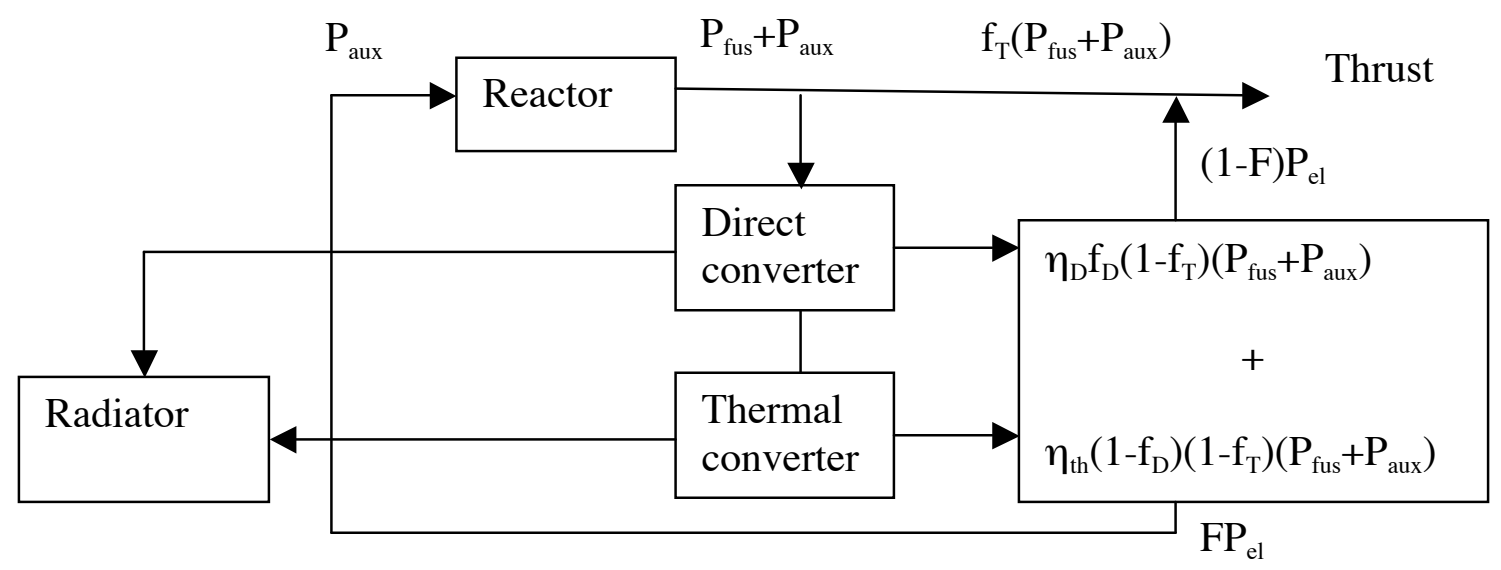

Fig.2.4 Idealized power flow 
A certain fraction of this power must be used for the auxiliary systems. If the efficiency for auxiliary power generation is $\eta_{\text {aux }}$, such a fraction is given by $\mathrm{P}_{\text {aux }} / \eta_{\text {aux }} \equiv F P_{\text {el }}$, with $F$ being the re-circulating power fraction.

The fusion gain $Q$ can then be related to $F, \eta_{\text {th }}$ and $\eta_{\text {aux }}$ by

$$
\mathrm{Q}=1 /\left(\mathrm{F} \eta_{\text {aux }}\left[\eta_{\mathrm{D}} \mathrm{f}_{\mathrm{D}}+\eta_{\mathrm{th}}\left(1-\mathrm{f}_{\mathrm{D}}\right)\right]\left(1-\mathrm{f}_{\mathrm{T}}\right)\right)-1
$$

The waste power to be radiated in space is therefore

$$
P_{\text {rad }}=\left[f_{D}\left(1-\eta_{D}\right)+\left(1-\eta_{\text {th }}\right)\left(1-f_{D}\right)\right]\left(1-f_{T}\right)\left(P_{\text {fus }}+P_{\text {aux }}\right)+\left(1-\eta_{\text {aux }}\right) P_{\text {aux }} / \eta_{\text {aux }}
$$

If the reactor is self-sustained $\left(\mathrm{P}_{\text {aux }}=0\right)$ then the re-circulating fraction vanishes. In practice this does not even occur for $\mathrm{P}_{\text {aux }}=0$ since part of the electric power must feed the control system, the cryogenic system, etc. Assuming the realistic value $\mathrm{F}=20 \%$ and $50 \%$ for both efficiencies, values of $\mathrm{Q}$ in the range $\mathrm{Q}=20-30$ are necessary for efficient energy production.

From the above expressions the power available for thrust is

$$
\mathrm{P}_{\text {thrust }}=\left[(1-\mathrm{F})\left[\eta_{\mathrm{D}} \mathrm{f}_{\mathrm{D}}+\eta_{\text {th }}\left(1-\mathrm{f}_{\mathrm{D}}\right)\right]\left(1-\mathrm{f}_{\mathrm{T}}\right)+\mathrm{f}_{\mathrm{T}}\right](1+1 / \mathrm{Q}) \mathrm{P}_{\text {fus }}
$$

\subsection{Mass budget}

In the following we consider the contribution to the mass arising from the various components.

\subsubsection{Radiator}

The waste power can be produced by the neutron power deposited in the blanket, by radiation and by the auxiliary systems. Energy is radiated in space following the StefanBoltzmann law

$$
\mathrm{P}_{\mathrm{rad}}=\varepsilon \sigma \mathrm{T}_{\mathrm{R}}^{4} \mathrm{~S}_{\mathrm{rad}}
$$

With $\varepsilon$ the radiator emissivity, $\sigma$ the Stefan-Boltzmann constant, $T_{R}$ the radiator temperature and $S_{\text {rad }}$ the radiator surface. It is apparent from the above expression that at fixed $\mathrm{P}_{\text {rad }}$, the radiator surface decreases as the radiator temperature increases. As shown in Roth (1989), the radiator temperature that minimizes the radiator mass in the limiting case of an ideal Carnot efficiency $\left(\eta=1-T_{R} / T_{H}\right)$ corresponds to $3 / 4$ of the temperature $T_{H}$ in the blanket/exhaust system. This estimate yields low values of the conversion efficiency $(\eta=25 \%)$. Present structural materials limits do not allow to go beyond $\mathrm{T}_{\mathrm{H}} \approx 300 \mathrm{C}$. The use of advanced materials (e.g. $\mathrm{SiC} / \mathrm{SiC}$ ) could achieve $\mathrm{T}_{\mathrm{H}} \approx 1000 \mathrm{C}$. If $\rho_{\mathrm{rad}}$ is the mass per unit surface (measured in $\mathrm{kg} / \mathrm{m}^{2}$ ) of the radiator, the radiator mass $\mathrm{M}_{\text {rad }}$ is linked to the fusion power by the following relation obtained by combining Eqs. (2.4) and (2.6) and using the Carnot expression for the efficiency 


$$
\mathrm{M}_{\mathrm{rad}}=\rho_{\text {rad }}\left\{\left[\mathrm{f}_{\mathrm{D}}\left(1-\eta_{\mathrm{D}}\right)+\left(\mathrm{T}_{\mathrm{R}} / \mathrm{T}_{\mathrm{H}}\right)\left(1-\mathrm{f}_{\mathrm{D}}\right)\right]\left(1-\mathrm{f}_{\mathrm{T}}\right)(1+1 / \mathrm{Q})+\left(1-\eta_{\text {aux }}\right) /\left(\mathrm{Q} \eta_{\text {aux }}\right)\right\} \mathrm{P}_{\text {fus }} / \varepsilon \sigma \mathrm{T}_{\mathrm{R}}^{4} \equiv \mathrm{P}_{\text {fus }} / \alpha_{\text {rad }}
$$

The above expression determines the specific power associated with the radiator. A reasonable value is $5 \mathrm{~kW}$ of rejected power for each $\mathrm{kg}$ of radiator mass, corresponding to $\rho_{\mathrm{rad}}=1.5 \mathrm{~kg} / \mathrm{m}^{2}$ and a radiating temperature of $600 \mathrm{~K}$. This type of numbers tend to be on the conservative side, as modern heat exchangers have specific weights of order 0.01 to $0.15 \mathrm{~kg} / \mathrm{kW}$.

\subsubsection{Magnet}

Present magnetic confinement concepts require the generation of magnetic fields in the range 1-10T in the plasma. Two different technologies are considered here: lowtemperature superconductors and actively cooled copper. The development of high temperature superconductors is still at a very preliminary stage to allow predicting the parameters of a fusion relevant system (see Casali 2004).

Superconductor technology. The development of low temperature superconductors for the International Tokamak Experimental Reactor (ITER) has currently produced $\mathrm{Nb}_{3} \mathrm{Sn}$ cables that can carry a current density in the range $50 \mathrm{MA} / \mathrm{m}^{2}$ at a magnetic field of $12.5 \mathrm{~T}$ (Huguet (2002)). The achievable current density of the cable depends on the strand performance (in the case of ITER $650 \mathrm{~A} / \mathrm{mm}^{2}$ ) but also on other parameters such as the $\mathrm{Cu} /$ non $\mathrm{Cu}$ ratio, the void fraction and the amount of space needed for the cooling channel, jacket and insulator which typically reduce the strand performance by an order of magnitude. Note however that $\mathrm{Nb} 3 \mathrm{Sn}$ strand with a critical current density of $2000 \mathrm{~A} / \mathrm{mm}^{2}$ have been already produced and that strand with a critical current density in the range of $3000 \mathrm{~A} / \mathrm{mm}^{2}$ are expected in the near future, about a factor three larger than the ITER requirements. Note also that the number above refer to a maximum magnetic field on the conductor of 12.5T: higher values of the critical current can be achieved at lower magnetic fields. Thus, values up to $250 \mathrm{MA} / \mathrm{m}^{2}$, envisaged in some study, can be considered already realistic. The cable specific weight assumed here is $6 \mathrm{t} / \mathrm{m}^{3}$ (using current tokamak magnetic practice and technology). A cylindrical solenoid with a radial width of $0.2 \mathrm{~m}$ can therefore produce a $12.5 \mathrm{~T}$ magnetic field. If $\mathrm{r}_{\mathrm{m}}$ and $\mathrm{V}$ are the radius of the solenoid and the internal volume, the mass of the magnet (neglecting the supporting structure) is approximately given by

$$
\mathrm{M}_{\mathrm{mag}} \approx 2.4 \mathrm{t}(\mathrm{B}(\mathrm{T}) / 12.5) \mathrm{V}\left(\mathrm{m}^{3}\right) / \mathrm{r}_{\mathrm{m}}(\mathrm{m})
$$

Actively-cooled copper magnet. The use of copper magnet technologies allows the achievement of larger magnetic fields, which, as we will see, lead to higher values of fusion power density. An upper bound to the magnet mass is given by the virial theorem

$$
\mathrm{M}_{\mathrm{mag}} \approx 2 \rho_{\mathrm{mag}}\left(\mathrm{B}^{2} / 2 \mu_{\mathrm{o}}\right) \mathrm{V} / \sigma_{\text {stress }}
$$


with $\sigma_{\text {stress }} \approx 1 \mathrm{GPa}$. Taking $\rho_{\mathrm{mag}}=2.5 \mathrm{t} / \mathrm{m}^{3}$ the above estimate yields about $600 \mathrm{t}$ for an ITER size magnet.

The magnet mass is proportional to the volume of the solenoid. Since within the present model the plasma volume $\mathrm{V}_{\mathrm{p}}$ is a factor $\left(\mathrm{r}_{\mathrm{p}} / \mathrm{r}_{\mathrm{m}}\right)^{2}$ smaller than the magnet volume and since the plasma volume is related to the fusion power by $\mathrm{P}_{\text {fus }}=\mathrm{P}_{\text {spec }} \mathrm{V}_{\mathrm{p}}$, with $\mathrm{P}_{\text {spec }}$ being the fusion power density in the reaction chamber, we can write

$$
M_{\text {mag }}=k_{m} V=k_{m}\left(r_{m} / r_{p}\right)^{2} P_{\text {fus }} / P_{\text {spec }} \equiv P_{\text {fus }} / \alpha_{\text {mag }}
$$

with $\mathrm{k}_{\mathrm{m}}$ given by Eq.(2.8) or (2.9).

The comparison between superconducting and copper magnet for fusion application shows that the use of superconductors gives always advantages in terms of the magnet mass over the copper magnets unless very-high magnetic field values are required.

\subsubsection{Cryoplant.}

Following Santarius (1998), a value for the mass per unit heat pumped of $1000 \mathrm{~kg} / \mathrm{kW}$ is assumed (one order of magnitude lower than the presently available systems). The cryoplant power is determined by the nuclear heating of the magnet:

$$
\mathrm{P}_{\text {cryo }}=\mathrm{f}_{\mathrm{n}} \mathrm{P}_{\text {fus }}\left(\mathrm{r}_{\mathrm{p}} / \mathrm{r}_{\mathrm{m}}\right) \exp \left(-\left(\mathrm{r}_{\mathrm{m}}-\mathrm{r}_{\mathrm{p}}\right) / \lambda_{\mathrm{n}}\right)
$$

with $\lambda_{\mathrm{n}} \approx 0.13 \mathrm{~m}$ being the neutron mean-free-path in the blanket and $\mathrm{f}_{\mathrm{n}}$ the fraction of fusion energy associated with neutrons.

The cryo-plant mass is therefore given by

$$
M_{\text {cryo }}=\mathrm{f}_{\mathrm{n}} \mathrm{P}_{\text {fus }}\left(\mathrm{r}_{\mathrm{p}} / \mathrm{r}_{\mathrm{m}}\right) \exp \left(-\left(\mathrm{r}_{\mathrm{m}}-\mathrm{r}_{\mathrm{p}}\right) / \lambda_{\mathrm{n}}\right) \times 10^{3} \mathrm{~kg} / \mathrm{kW} \equiv \mathrm{P}_{\text {fus }} / \alpha_{\text {cryo }}
$$

\subsubsection{Blanket}

An optimized blanket made by $\mathrm{LiH}$ has been proposed in Kulcinski (1987) with a density $\rho_{\mathrm{s}}$ in the range $10^{3} \mathrm{~kg} / \mathrm{m}^{3}$ (value used by Santarius (1998)) and much less than the values around $10^{4} \mathrm{~kg} / \mathrm{m}^{3}$ of the solid and liquid blankets envisaged for a fusion reactor. The associated mass is given by

$$
\mathrm{M}_{\mathrm{s}}=\rho_{\mathrm{s}}\left(1-\mathrm{r}_{\mathrm{p}}^{2} / \mathrm{r}_{\mathrm{m}}{ }^{2}\right) \mathrm{V}=\rho_{\mathrm{s}}\left(\mathrm{r}_{\mathrm{m}}{ }^{2} / \mathrm{r}_{\mathrm{p}}{ }^{2}-1\right) \mathrm{P}_{\mathrm{fus}} / \mathrm{P}_{\mathrm{spec}} \equiv \mathrm{P}_{\text {fus }} / \alpha_{\mathrm{s}}
$$

\subsubsection{Auxiliary systems}

The estimate used in Williams (1998) for the negative neutral beam system correspond to an efficiency of $29 \%$ (108MW beam power out of $367 \mathrm{MW}$ input power). The total mass is dominated by the 20 sources $(2.5 \mathrm{t}$ each) which include the filament source, the three stage accelerator and the neutralizer. These assumptions correspond to a reduction by about an order of magnitude of the existing systems. Much lower mass estimates have 
been used by Cheung (2004). We take here a figure of $2.5 \mathrm{~kg} / \mathrm{kW}$ of injected power. The mass of the auxiliary system is given by

$$
\mathrm{M}_{\text {aux }}=2.5 \mathrm{~kg} / \mathrm{kW} \mathrm{P}_{\text {fus }} / \mathrm{Q}
$$

Since $\mathrm{Q} \approx 20$ we neglect in the following this contribution.

\subsubsection{Conversion.}

A high efficiency closed Brayton thermodynamic cycle is envisaged. The working fluid to transport heat is typically He. The mass budget for a $400 \mathrm{MWe}$ system $(20 \%$ efficiency) operating with an inlet temperature of $1700 \mathrm{~K}$ and outlet temperature of $1300 \mathrm{~K}$ (see Williams (1998)) is about 145t. In Cheung (2004) an efficiency of $40 \%$ is used (7MW produced out of an input of $18 \mathrm{MW}$ ). The mass was calculated using a figure of $3 \mathrm{~kg} / \mathrm{kWe}$ for the conversion system (excluding the radiator). As is the case of other figures cited in such calculations at times such figures are either strongly underestimated or, as in this particular case, widely overestimated. For instance, at $3 \mathrm{~kg} / \mathrm{kWe}$, the total of the $400 \mathrm{MWe}$ system would be 1200t. In our simplified analysis we neglect this component.

\subsection{Specific power}

By adding all the contributions coming from the above expressions it is possible to evaluate an expression for the specific power.

$$
\mathrm{M} \equiv \mathrm{P}_{\text {thrust }} / \alpha=\mathrm{P}_{\text {fus }}\left(1 / \alpha_{\mathrm{mag}}+1 / \alpha_{\mathrm{s}}+1 / \alpha_{\text {cryo }}+1 / \alpha_{\text {rad }}\right)
$$

Upon substituting all the various expressions derived above, we obtain

$$
\begin{aligned}
\alpha(\mathrm{kW} / \mathrm{kg})=[ & \left.(1-\mathrm{F})\left[\eta_{\mathrm{D}} \mathrm{f}_{\mathrm{D}}+\eta_{\mathrm{th}}\left(1-\mathrm{f}_{\mathrm{D}}\right)\right]\left(1-\mathrm{f}_{\mathrm{T}}\right)+\mathrm{f}_{\mathrm{T}}\right](1+1 / \mathrm{Q}) \times \\
& \left\{\left(\mathrm{r}_{\mathrm{m}} / \mathrm{r}_{\mathrm{p}}\right)^{2} \mathrm{P}_{\text {spec }}\left(\mathrm{MW} / \mathrm{m}^{3}\right)^{-1}\left[\mathrm{k}_{\mathrm{m}}+\left(1-\mathrm{r}_{\mathrm{p}}{ }^{2} / \mathrm{r}_{\mathrm{m}}{ }^{2}\right) \rho_{\mathrm{s}}\left(\mathrm{t} / \mathrm{m}^{3}\right)\right]+\right. \\
& +\mathrm{f}_{\mathrm{n}} 10^{3}\left(\mathrm{r}_{\mathrm{p}} / \mathrm{r}_{\mathrm{m}}\right) \exp \left[-\left(\mathrm{r}_{\mathrm{m}}-\mathrm{r}_{\mathrm{p}}\right) / \lambda_{\mathrm{n}}\right]+ \\
& \left.+\left[\mathrm{f}_{\mathrm{D}}\left(1-\eta_{\mathrm{D}}\right)+\left(1-\eta_{\text {th }}\right)\left(1-\mathrm{f}_{\mathrm{D}}\right)\right]\left(1-\mathrm{f}_{\mathrm{T}}\right)(1+1 / \mathrm{Q})+\left(1-\eta_{\text {aux }}\right) /\left(\mathrm{Q} \eta_{\text {aux }}\right)\right] \\
& \left.\times 10^{3} \rho_{\text {rad }}\left[\varepsilon \sigma \mathrm{T}_{\mathrm{R}}^{4}\right]^{-1}\right\}^{-1}
\end{aligned}
$$

with $\mathrm{k}_{\mathrm{m}}=2.4(\mathrm{~B}(\mathrm{~T}) / 12.5) \mathrm{r}_{\mathrm{m}}(\mathrm{m})^{-1}$ for the case of superconducting magnet technology and $\mathrm{k}_{\mathrm{m}}=2 \times 10^{-3} \mathrm{~B}(\mathrm{~T})^{2}$ for the case of copper technology.

The simplest limiting case for the above expression is $f_{n}=0$ (aneutronic reactions) and $\mathrm{f}_{\mathrm{T}}=1$ (direct propulsion) which yields (with $\mathrm{r}_{\mathrm{p}}=\mathrm{r}_{\mathrm{m}}$, i.e. no shield)

$$
\alpha(\mathrm{kW} / \mathrm{kg}) \approx \mathrm{P}_{\mathrm{spec}}\left(\mathrm{MW} / \mathrm{m}^{3}\right) / \mathrm{k}_{\mathrm{m}}
$$

Thus, to obtain specific power values in the range of $1-10 \mathrm{~kW} / \mathrm{kg}$ the fusion power density for aneutronic reactions must be in the range $1-10 \mathrm{MW} / \mathrm{m}^{3}$ times the constant $\mathrm{k}_{\mathrm{m}} \approx 1$.

In the case of neutron producing reactions, it is convenient first to maximize Eq.(2.15) with respect to the ratio $r_{p} / r_{m}$ (at fixed $r_{m}$ ) (to minimize the cryoplant plus blanket mass) 
and then with respect to the ratio $T_{R} / T_{H}$ (assuming the Carnot expression for the efficiency $\eta$ ) at fixed $\mathrm{T}_{\mathrm{H}}$ (determined by the structural material limitations).

Two limiting cases can be singled out depending on whether the radiator mass or the fusion system mass tend to dominate:

Case a: large radiator mass $\left(\varepsilon \sigma \mathrm{T}_{\mathrm{R}}{ }^{4} / \rho_{\mathrm{rad}}<<\left(\mathrm{r}_{\mathrm{p}} / \mathrm{r}_{\mathrm{m}}\right)^{2} \mathrm{P}_{\text {spec }}\left(\mathrm{MW} / \mathrm{m}^{3}\right) /\left(\mathrm{k}_{\mathrm{m}}+\rho_{\mathrm{s}}\left(\mathrm{t} / \mathrm{m}^{3}\right)\right)\right)$.

In this limit the mass budget is dominated by the radiator and the specific power is independent of the fusion power density

$$
\begin{array}{r}
\alpha(\mathrm{kW} / \mathrm{kg}) \approx\left[\varepsilon \sigma \mathrm{T}_{\mathrm{R}}^{4}\right] /\left(10^{3} \rho_{\mathrm{rad}}\right)\left[(1-\mathrm{F})\left[\eta_{\mathrm{D}} \mathrm{f}_{\mathrm{D}}+\eta_{\text {th }}\left(1-\mathrm{f}_{\mathrm{D}}\right)\right]\left(1-\mathrm{f}_{\mathrm{T}}\right)+\mathrm{f}_{\mathrm{T}}\right](1+1 / \mathrm{Q}) \times \\
{\left[\left[\mathrm{f}_{\mathrm{D}}\left(1-\eta_{\mathrm{D}}\right)+\left(1-\eta_{\text {th }}\right)\left(1-\mathrm{f}_{\mathrm{D}}\right)\right]\left(1-\mathrm{f}_{\mathrm{T}}\right)(1+1 / \mathrm{Q})+\left(1-\eta_{\text {aux }}\right) /\left(\mathrm{Q} \eta_{\text {aux }}\right)\right]^{-1}}
\end{array}
$$

The radiator temperature reduces to $\mathrm{T}_{\mathrm{R}}=3 / 4 \mathrm{~T}_{\mathrm{H}}$ in the limit $\mathrm{f}_{\mathrm{D}}<<1, \mathrm{f}_{\mathrm{T}}<<1$ (see Roth (1989)). Note that the radiator temperature can become larger than the blanket temperature $T_{H}$ for finite values of $f_{T}$ and $f_{D}$ : this result simply means that if the fraction of energy going directly to thrust or recovered by direct electricity conversion is large, there is no need to have thermal electricity conversion and the remaining fraction must be radiated at the highest possible temperature. For a radiator able to radiate $5 \mathrm{~kW} / \mathrm{kg}$, Eq.(2.17) predicts a specific power in the range from $1 \mathrm{~kW} / \mathrm{kg}$ (for $\mathrm{f}_{\mathrm{D}}=\mathrm{f}_{\mathrm{T}}=0$, i.e. fusion electric propulsion) to $9 \mathrm{~kW} / \mathrm{kg}$ (for $\mathrm{f}_{\mathrm{D}}=\mathrm{f}_{\mathrm{T}}=0.5$, in which only $25 \%$ of the power must be radiated). The specific power increases very rapidly as $f_{D}$ and $f_{T}$ increase. It is apparent that fusion electric propulsion is marginal in terms of specific power. Note that Eq.(2.17) is independent of any parameter related to the plasma behavior.

Case b: small radiator mass $\left(\varepsilon \sigma \mathrm{T}_{\mathrm{R}}^{4} / \rho_{\mathrm{rad}}>\left(\mathrm{r}_{\mathrm{p}} / \mathrm{r}_{\mathrm{m}}\right)^{2} \mathrm{P}_{\text {spec }}\left(\mathrm{MW} / \mathrm{m}^{3}\right) /\left(\mathrm{k}_{\mathrm{m}}+\rho_{\mathrm{s}}\left(\mathrm{t} / \mathrm{m}^{3}\right)\right)\right)$.

In this limit the radiator mass is negligible with respect to the reactor mass

$$
\left.\alpha(\mathrm{kW} / \mathrm{kg}) \approx\left[(1-\mathrm{F}) \eta_{\mathrm{D}} \mathrm{f}_{\mathrm{D}}+\eta_{\mathrm{th}}\left(1-\mathrm{f}_{\mathrm{D}}\right)\right]\left(1-\mathrm{f}_{\mathrm{T}}\right)+\mathrm{f}_{\mathrm{T}}\right](1+1 / \mathrm{Q})\left(\mathrm{r}_{\mathrm{p}} / \mathrm{r}_{\mathrm{m}}\right)^{2} \mathrm{P}_{\mathrm{spec}}\left(\mathrm{MW} / \mathrm{m}^{3}\right) /\left(\mathrm{k}_{\mathrm{m}}+\rho_{\mathrm{s}}\left(\mathrm{t} / \mathrm{m}^{3}\right)\right)
$$

with

$$
\mathrm{r}_{\mathrm{m}}=\mathrm{r}_{\mathrm{p}}+3 \lambda_{\mathrm{n}} \ln 10-\lambda_{\mathrm{n}} \ln \left\{2\left(\mathrm{r}_{\mathrm{m}} / \mathrm{r}_{\mathrm{p}}\right)^{3}\left(\mathrm{k}_{\mathrm{m}}+\rho_{\mathrm{s}}\right) /\left[\mathrm{f}_{\mathrm{n}} \mathrm{P}_{\mathrm{spec}}\left(\mathrm{MW} / \mathrm{m}^{3}\right)\left(\mathrm{r}_{\mathrm{p}} / \lambda_{\mathrm{n}}+1\right)\right]\right\}
$$

This solution is a generalization of Eq.(2.16) to include the blanket mass. The radiator temperature can now be substantially lower than $T_{H}$ and high values of the efficiency $\eta$ can be obtained. For a radiator able to radiate $5 \mathrm{~kW} / \mathrm{kg}$ (as noted, a conservative value), Eq.(2.18) becomes valid for

$$
\begin{aligned}
& \left\{\left[\mathrm{f}_{\mathrm{D}}\left(1-\eta_{\mathrm{D}}\right)+\left(1-\eta_{\text {th }}\right)\left(1-\mathrm{f}_{\mathrm{D}}\right)\right]\left(1-\mathrm{f}_{\mathrm{T}}\right)(1+1 / \mathrm{Q})+\left(1-\eta_{\text {aux }}\right) /\left(\mathrm{Q} \eta_{\text {aux }}\right)\right\} \quad \mathrm{P}_{\text {spec }}\left(\mathrm{MW} / \mathrm{m}^{3}\right) \quad< \\
& \left(\mathrm{r}_{\mathrm{m}} / \mathrm{r}_{\mathrm{p}}\right)^{2}\left(\mathrm{k}_{\mathrm{m}}+\rho_{\mathrm{s}}\left(\mathrm{t} / \mathrm{m}^{3}\right)\right)
\end{aligned}
$$

\subsection{Fusion power density}

In order to understand which values of the specific power can be expected from a fusion reactor and how are they related with the plasma parameters, it is convenient to assume 
that the operating temperature is close to the optimal temperature $T_{\text {opt }}$ (i.e. the temperature corresponding to the minimum of the $n \tau$ vs. T curve). The optimal temperature depends on the reaction chosen, on the gain $\mathrm{Q}$ and on the radial profile factors. Then the electron density can be expressed in terms of the parameter beta $\left(\beta \equiv 2 \mu_{\mathrm{o}} \mathrm{f}_{1} \mathrm{n}_{\mathrm{e}} \mathrm{T} / \mathrm{B}^{2}\right.$, with $\mathrm{f}_{1} \equiv 1+\Sigma_{\mathrm{i}} \mathrm{n}_{\mathrm{i}} / \mathrm{n}_{\mathrm{e}}$, a factor of order unity depending on the fuel composition)

$$
\mathrm{n}_{\mathrm{e}}=\beta \mathrm{B}^{2} /\left(2 \mu_{\mathrm{o}} \mathrm{f}_{\mathbf{1}} \mathrm{T}_{\mathrm{opt}}\right)
$$

The achievable values of $\beta$ depend on the stability properties of the specific magnetic configuration considered and will be discussed in the next section. Note that to express the plasma density in terms of $\beta$ is correct as long as no more stringent limits on the plasma density are discovered (e.g., in tokamak operation density is observed to reach a maximum experimentally proportional to the average plasma current density).

From the above conditions, it is possible to determine the fusion power per unit volume that can be produced in the form of neutrons and charged particles

$$
\mathrm{P}_{\text {spec }}=\mathrm{n}^{2} \mathrm{f}_{2}<\sigma v>\mathrm{E}_{\text {fus }}=\left(\beta \mathrm{B}^{2} /\left(2 \mu_{\mathrm{o}} \mathrm{f}_{1} \mathrm{~T}_{\text {opt }}\right)\right)^{2} \mathrm{f}_{2}<\sigma v>\mathrm{E}_{\text {fus }}
$$

with $\mathrm{f}_{2} \equiv\left(\mathrm{n}_{\mathrm{i}} / \mathrm{n}_{\mathrm{e}}\right)\left(\mathrm{n}_{\mathrm{j}} / \mathrm{n}_{\mathrm{e}}\right)$ a coefficient related to the fuel composition and $\mathrm{E}_{\text {fus }}$ the energy released in a fusion reaction. It is apparent that in order to maximize the fusion power density, for a given reaction the plasma density must achieve the largest possible value. From Eq.(2.3) this can be accomplished both by maximizing the value of beta and by operating at large values of the magnetic field.

For the sake of illustration, in the following Table the values of $\mathrm{P}_{\text {spec }}$ achievable with the DT and the $\mathrm{D}^{3} \mathrm{He}$ reactions is shown for three different values of $\beta$ and a magnetic field of $10 \mathrm{~T}$.

\begin{tabular}{|l|l|l|}
\hline & DT & $\mathrm{D}^{3} \mathrm{He}$ \\
\hline$\beta=100 \%$ & $10^{4} \mathrm{MW} / \mathrm{m}^{3}$ & $123 \mathrm{MW} / \mathrm{m}^{3}$ \\
\hline$\beta=10 \%$ & $10^{2} \mathrm{MW} / \mathrm{m}^{3}$ & $1.2 \mathrm{MW} / \mathrm{m}^{3}$ \\
\hline$\beta=1 \%$ & $1 \mathrm{MW} / \mathrm{m}^{3}$ & $0.01 \mathrm{MW} / \mathrm{m}^{3}$ \\
\hline
\end{tabular}

Table 2.2 Fusion power per unit volume

If we compare the DT and the $\mathrm{D}^{3} \mathrm{He}$ reactions, at the same value of $\left(\beta \mathrm{B}^{2}\right)$, it follows that the $\mathrm{D}^{3} \mathrm{He}$ reaction has a specific power about two orders of magnitude lower than that of the DT reaction. Table 2.2 clearly shows that the use of the $\mathrm{D}^{3} \mathrm{He}$ reaction becomes interesting only if values of $\beta$ above $10 \%$ can be achieved.

It should be noted that a limit exists to the neutron power $\mathrm{P}_{\mathrm{n}}$ per unit surfaces that can be tolerated by the first wall before serious degradation of the structural properties. For fusion reactor application, the target specific fluence is $P_{n} \Delta T / S \approx 10-15 M W y / m^{2}$. This value depends on the neutron energy (with the $14 \mathrm{MeV}$ of the DT reaction being the worse situation). The target for first wall replacement is 5 years at full power. This set a limit to $2-3 \mathrm{MW} / \mathrm{m} 2$ for the specific neutron power. The specific neutron power depends 
on the shape of the reaction chamber. In the case of a spherical chamber of radius $r_{w}$ it is given by $P_{n} / S=f_{n} P_{\text {spec }} r_{w} / 3$. For a cylindrical chamber of radius $r_{w}$ and length $L$ is given by $\mathrm{P}_{\mathrm{n}} / \mathrm{S}=\mathrm{f}_{\mathrm{n}} \mathrm{P}_{\text {spec }} \mathrm{r}_{\mathrm{w}} / 2$. Thus, the limit on the neutron wall load imposes a limit on the specific power that is more stringent for large chamber radii. Taking as an example 1 year of full power operation, the specific power would be limited by

$$
\mathrm{P}_{\mathrm{spec}}\left(\mathrm{MW} / \mathrm{m}^{3}\right)<20-45 /\left(\mathrm{f}_{\mathrm{n}} \mathrm{r}_{\mathrm{w}}(\mathrm{m})\right)
$$

\subsection{Summary}

It is convenient to summarize at this point the results of the analysis presented under all the assumption made.

- If the system mass is dominated by the radiator, the specific power $\alpha$ does not depend on the fusion power per unit volume and, using the conservative assumption that $5 \mathrm{~kW}$ can be radiated per each $\mathrm{kg}$ of radiator mass, $\alpha$ can vary between $1 \mathrm{~kW} / \mathrm{kg}$ in the case of pure fusion electric propulsion to $10 \mathrm{~kW} / \mathrm{kg}$ if the fuel kinetics permits conversion of $50 \%$ of the fusion power to thrust power.

- If the reactor mass dominates, the specific power increases linearly with fusion power density. Fusion power density in excess of $1 \mathrm{MW} / \mathrm{m}^{3}$ is needed. This is compatible with the use of advanced fuels such as $\mathrm{D}^{3} \mathrm{He}$ only if values of $\beta$ above $10 \%$ can be achieved.

- The fusion power density cannot exceed the value given in Eq.2.22 (which assumes $1 \mathrm{yr}$ of full power operation) due to the constraint on the neutron wall load. 


\section{Status of open magnetic field configuration research}

3.1 Classification and present status of open magnetic field configurations.

It has been shown in the previous section that in order to achieve large specific power it is necessary to use to the largest possible extent fusion in the form of the direct propulsion with the possibility of direct electricity conversion. This is not easy to achieve in equilibrium configurations, such as conventional tokamaks, where plasma does not escape from the reaction chamber, but could be achieved by open magnetic field (OMF) configurations. The topology of OMF configuration can be very different: mirrors topology is cylindrical, field reversed configurations and spheromaks transition to a torus in the confinement region. Nevertheless, the common feature of open magnetic field configuration is that the magnetic field lines escape from the plasma confinement zone without intercepting any wall, and such feature enables using the fusion plasma both for direct propulsion and direct conversion. Note that such a feature may be common also to other systems such as the very low aspect ratio (spherical) tokamak, not considered here but already proposed for fusion propulsion applications.

The best plasma performance achieved so far has been obtained in closed magnetic field configurations (specifically, in tokamaks). However, open magnetic field configurations have intrinsic advantages:

- easy steady state operation;

- natural particle exhaust;

- $\quad$ high $\beta$ (=thermal pressure/magnetic pressure);

- simple design;

- simple application to space propulsion since they allow to convert the fusion power directly into thrust.

In the following, we consider three main classes of OMF configurations:

- Open ended systems such as mirrors;

- Closed field line systems such as field reversed configurations (FRC) and spheromaks;

- Levitated dipoles.

The analysis below addresses the potential of these configurations to achieve high- $\beta$ values, which is mandatory for the use of advanced fuels, and good confinement properties (i.e. large $n \tau$ values and reasonable fusion gain) under conditions typical of sustained thrust production. To fully assess the potential of a configuration requires a good theoretical understanding of the underlying physical processes. Unfortunately this is not possible in all the cases. In some limiting cases the answer provided by the experimental evidence obtained so far may be enough to draw a conclusion about the extrapolability of the results to a range of parameters relevant for a burning plasma. This is the case of ideal MHD stability, where the stability of a given magnetic configuration depends only by the shape of the magnetic fields and on $\beta$. However, weaker MHD modes are heavily affected by kinetic effects related, for instance, to finite particle orbits width. In some cases even the application of the ideal MHD model is questionable due to the large orbit size in some of the configuration examined below. In order to understand 
the distance between the proposed configuration and the existing devices we consider in the following three dimensionless parameters:

- $\quad \beta$, the ratio between the plasma pressure and magnetic pressure;

- the collisionality parameter (usually indicated by $v_{*}$ ) defined as the ratio between the typical scale length along the magnetic field and the mean free path for Coulomb collision;

- the normalized Larmor radius $\rho_{*}$ defined as the ratio between the ion Larmor radius and the typical scale length transverse to the magnetic field.

It can indeed be easily shown (see e.g. Kadomtsev (1975)) that the plasma physics equation (i.e. the Boltzmann plus Maxwell equations) can be cast in a dimensionless form and, if the Debye length does not play any role in the processes underlying stability and transport (which is always the case) full equivalence between plasma behavior is assured by identical values of the three dimensionless parameters defined above. For comparison, present tokamak experiments have achieved values of $\beta$ and $\nu_{*}$ similar to those of interest for ITER and the extrapolation in $\rho_{*}$ is about a factor 3 .

\subsection{Mirror configurations.}

Mirror configurations confine the plasma in a solenoidal magnetic "bottle". They are a natural candidate for fusion propulsion since they allow the plasma to exhaust at one end of the "bottle", thus producing thrust, and, simultaneously, direct energy conversion (Kammash 1995a). The question that arises is: can mirror configurations achieve the fusion power density needed for space propulsion? In this context, a review of the present status of mirror research can be found in (Post 1987).

\subsubsection{The simple mirror configuration.}

At the simplest level, a mirror-configuration consists of a pair of Helmoltz-coils with currents flowing in the same direction as shown in Fig. 3.1.

The magnetic field intensity varies along $\mathbf{B}$ with a minimum value $B_{\min }$ at the middle and a maximum value $B_{\max }$ at the coil location. The confinement in the simplest mirror configuration arises from the conservation of the energy $E=m v^{2} / 2$ and of the first adiabatic invariant (the magnetic moment $\mu=\mathrm{mv}_{\perp}{ }^{2} / 2 \mathrm{~B}, \mathrm{v}_{\perp}$ being the particle velocity perpendicular to $\mathbf{B}$ ) of a particle of mass $m$ moving in a weakly inhomogeneous magnetic field $\mathbf{B}$. The conservation laws imply that a particle moving along the field (with velocity $\mathrm{V}_{\|}$) is reflected at the plasma location where $\mathrm{mv}_{\|}{ }^{2} / 2 \equiv \mathrm{E}-\mu \mathrm{B}=0$. Therefore, upon producing a magnetic field configuration such as that shown in Fig. 3.1, particles will be trapped provided that the ratio $\mu / \mathrm{E}$ is larger than $1 / \mathrm{B}_{\max }$. 


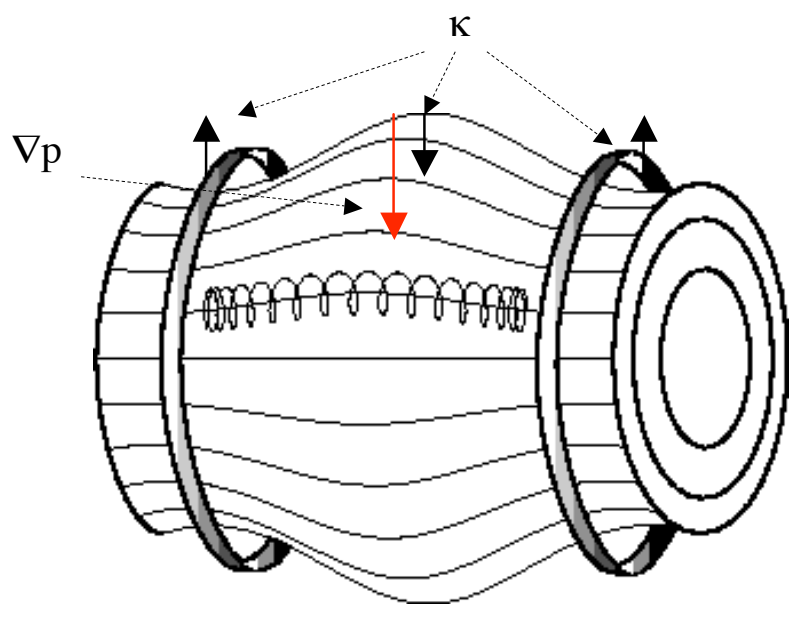

Fig.3.1 Simple mirror field configurations. The direction of the magnetic field curvature $\kappa$ is also shown.

It can be easily shown that in the case of an isotropic particle distribution function in the velocity space, the fraction $\mathrm{f}_{\mathrm{T}}$ of plasma particles satisfying the trapping condition is given by $\mathrm{f}_{\mathrm{T}} \approx(1-1 / \mathrm{R})^{1 / 2}$ with $\mathrm{R} \equiv \mathrm{B}_{\max } / \mathrm{B}_{\min }$, the so-called mirror ratio. Particles not satisfying this condition will be promptly lost, with the result of producing an anisotropic distribution function characterized by a "loss-cone" in velocity space. For large values of the mirror ratio, the fraction of unconfined particles is given by $1-f_{T} \approx 1 / 2 R$. Obviously, the fraction of unconfined particles can be made smaller if they are injected in the configurations with small parallel velocity, e.g. by perpendicular neutral beam injection. On the other hand, collisions tend to restore isotropy and the loss-cone is continuously populated by scattering in velocity space.

Since the electrons have a larger collision frequency than the ions, they are scattered in the loss-cone (and therefore lost) at a higher rate. As a consequence, the plasma tends to be positively charged. Its potential, $\phi$, is determined by the condition that transport must be ambipolar, i.e. that overall charge neutrality must be maintained, yielding values in the range $e \phi \approx 4-8 \mathrm{~T}_{\mathrm{e}}$. The effect of the ambipolar potential is that of decreasing the losses of low-energy electrons and increasing the losses of ions.

As a result, in such a simple configuration confinement is maintained on the ionion collision time scale $\tau_{\mathrm{ii}}$ (the time scale for the scattering of a trapped ion into the losscone). The ion-ion collision time is proportional to $\mathrm{E}_{\mathrm{i}}{ }^{3 / 2}$, with $\mathrm{E}_{\mathrm{i}}$ the ion energy, therefore higher values of the confinement are achieved by increasing $\mathrm{E}_{\mathrm{i}}$. On the other hand, fast ions tend to transfer their energy by Coulomb collision preferentially to electrons if $\mathrm{E}_{\mathrm{i}}>15 \mathrm{~T}_{\mathrm{e}}$. If the electron temperature is too low, the slowing-down of the injected ions by the electrons (electron drag) occurs on a fast time scale $\tau_{\mathrm{SD}} \propto \mathrm{T}_{\mathrm{e}}^{3 / 2} / \mathrm{n}_{\mathrm{e}}$. Thus, electrons must be kept at sufficiently high temperature.

To achieve high electron temperature in an open-ended configuration might appear at a first sight a very difficult task. Simple considerations based on classical fluid transport theory would predict very large electron thermal conduction (and therefore very high heating power to keep the electrons at a sufficiently high temperature). However, in experiments characterized by a low value of collisionality (i.e. a mean-free-path longer than the mirror distance), the electron thermal conductivity along the magnetic field is much lower than the classical estimate. This result is a consequence of the presence of the 
ambipolar potential $\phi$ that confines the electrons inside the mirror. Only the supra-thermal electrons can escape the barrier and contribute to thermal conduction. This has the effect of a dramatic reduction of the electron thermal conductivity.

The $n \tau$ parameter can be estimated by solving the Fokker-Planck equation accounting for the presence of the ambipolar potential and the electron drag. It can be shown (Post (1987)) that the confinement parameter is approximately given by

$$
\mathrm{n} \tau \approx 2.5 \times 10^{16} \mathrm{E}_{\mathrm{i}}(\mathrm{keV})^{3 / 2} \log _{10}(\mathrm{R}) \mathrm{m}^{-3} \mathrm{~s}
$$

Note that the dependence on the mirror ratio $\mathrm{R}$ is only logarithmic and that the above expression is independent of size and magnetic field. In order to obtain a significant gain, values of $E_{i}$ in the range of a few hundreds $\mathrm{keV}$ are needed. However, above a certain energy the fusion cross sections tend to decrease (at $100 \mathrm{keV}$ for the DT and $400 \mathrm{keV}$ for the $\mathrm{D}^{3} \mathrm{He}$ in the centre of mass frame) therefore an optimal value exists for the ion energy.

All these constraints limit efficient energy production by the simple mirror. Indeed, at the simplest level a mirror reactor works as an energy amplifier. Power is injected through high energy neutral beams and fusion power is recovered with a gain $\mathrm{Q} \equiv \mathrm{P}_{\text {fus }} / \mathrm{P}_{\text {inj }}$, with $\mathrm{Q}$ given by

$$
\mathrm{Q} \approx(\mathrm{n} \tau / 4)<\sigma v>\mathrm{E}_{\mathrm{fus}} / \mathrm{E}_{\mathrm{i}}
$$

with $\mathrm{E}_{\text {fus }}$ the energy released by the fusion reaction. The above $n \tau$ scaling implies for a simple-mirror configuration (using the DT reaction) with $\mathrm{R}=10, \mathrm{E}_{\mathrm{i}} \approx 300 \mathrm{keV}$ values of $\mathrm{Q}$ in the range $\mathrm{Q} \approx 1$, too low even employing advanced techniques for electricity production such as direct conversion. Even lower values $(\mathrm{Q} \approx 0.3)$ are obtained for the $\mathrm{D}-{ }^{3} \mathrm{He}$ reaction.

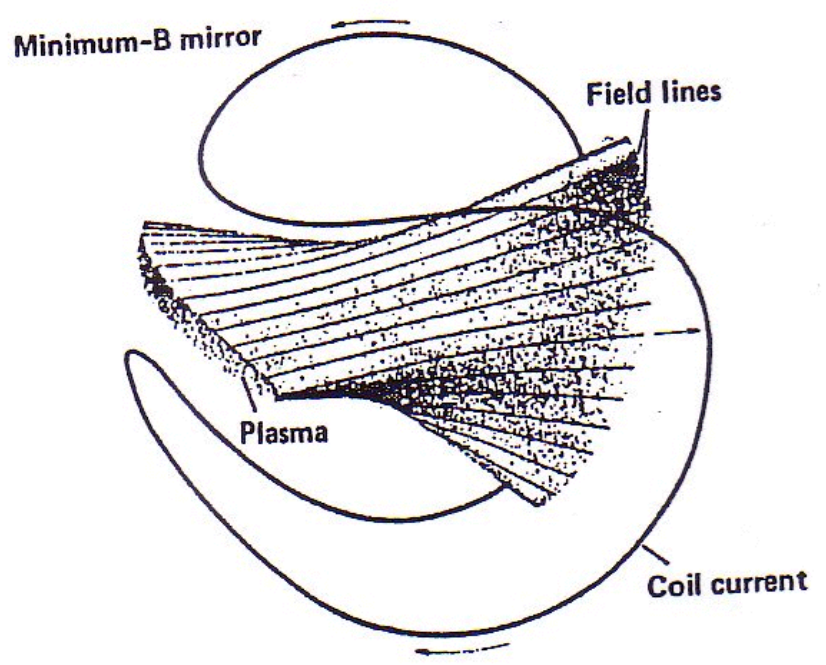

Fig.3.2 Baseball coils from Post (1987)

In addition to its low gain, the simple mirror configuration has limited MHD stability properties due to the presence of interchange instabilities in the region between the 
mirrors: indeed the exchange of a plasma flux tube with a vacuum flux tube is energetically favorable if the local magnetic field curvature $\mathbf{\kappa}(\mathbf{K} \equiv \mathbf{b} \cdot \nabla \mathbf{b}$, with $\mathbf{b} \equiv \mathbf{B} / \mathbf{B})$ is parallel to the pressure gradient, as in the central part of the mirror cell (the opposite occurs near the mirror points) (see Fig.3.1). The instability is suppressed by superimposing a multipolar field to produce a so-called "minimum-B" configuration in which a "magnetic well" is produced around the symmetry axis. The demonstration of the stability of minimum-B configurations was achieved in modified mirror systems called "baseball", or Ying-Yang, coils shown in Fig.3.2. Unfortunately, the breaking the axial symmetry, when a multipolar component is superimposed to the axisymmetric mirror field, has a detrimental effect on the radial particle transport: radial particle drifts are produced that cause increased transport losses either by collisions, as in closed toroidal magnetic configurations, or by resonant processes.

Small-scale instabilities can be also generated by the existence of anisotropies in velocity space and in particular of the loss cone. These instabilities have been shown to be much less deleterious than theoretically predicted, provided a warm plasma is injected into the mirror and will not be considered further (see Post 1987 for a discussion of the various microinstabilities in mirrors).

To overcome all these problems, advanced mirror concepts have been proposed, briefly reviewed in the rest of this section.

\subsubsection{The tandem mirror.}

The idea behind the Tandem Mirror (TM) is to modify the electrostatic potential shape along $\mathbf{B}$ in such a way as to confine both escaping ions and electrons. In the Tandem Mirror (Fig. 3.3) two smaller mirror cells are added at each end of the larger central cell where fusion reactions are supposed to take place.

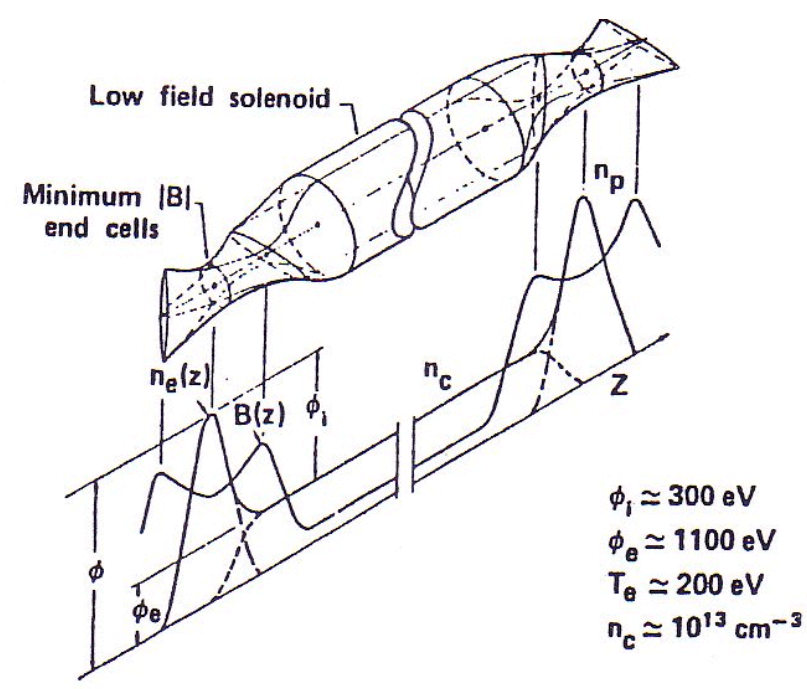

Fig.3.3 Tandem Mirror from Post (1987)

The density and temperature axial profiles in the two end cells are tailored, using external methods such as radiofrequency heating and neutral beam injection, to transform them in positive potential electrostatic "plugs" which reduce the losses of positive ions from the 
central cell. The axial profiles of density, temperature and electrostatic potential are shown in Fig. 3.4.

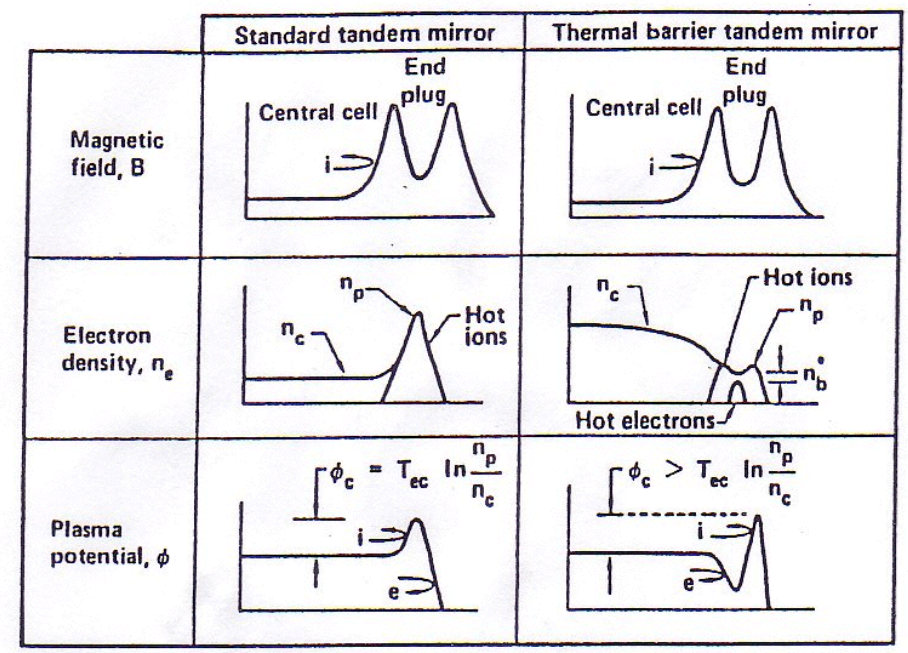

Fig. 3.4 Axial profiles in a Tandem Mirror. Schematic illustration from Post (1987) showing a comparison between density and electrostatic potential profile in a standard tandem mirror and in a tandem mirror with thermal barrier

The plasma potential, electron density and electron temperature are related by the condition that the highly mobile electrons relax to a Boltzmann distribution, yielding

$$
\phi(\mathrm{z})=\phi\left(\mathrm{z}_{\mathrm{o}}\right)+\mathrm{T}_{\mathrm{e}} \ln \left[\mathrm{n}(\mathrm{z}) / \mathrm{n}\left(\mathrm{z}_{\mathrm{o}}\right)\right]
$$

with $\mathrm{z}$ the axial coordinate and $\mathrm{z}_{\mathrm{o}}$ corresponding to the mid-plane. The above equation suggests two possible schemes of plasma potential tailoring:

- the conventional TM scheme, in which higher potential in the plug cells is achieved by increasing the plug density with respect to the central cell density; such an increase is obtained by energetic ion injection in the plug; the magnetic field, density and plasma potential axial variations are shown in Fig.3.4; since the density in the central cell must be sufficiently high to achieve large fusion density $\left(\mathrm{P}_{\text {fus }} \propto \mathrm{n}^{2}\right)$, very high values of the plug density must be achieved and this implies very high magnetic field $(\approx 15 \mathrm{~T})$ in the end cells and high-energy neutral beam injection $(\mathrm{E} \approx 1 \mathrm{MeV})$. Note that in this configuration the electrons are reflected at the end of the two plug-cells, therefore the electrons in the plug are in thermal contact with the electrons in the central cell: any attempt to increase the temperature in the plug will increase also the temperature in the central cell and therefore will increase the power demand;

- the thermal-barrier TM scheme, in which the electrons are reflected before reaching the central cell. This scheme thermally isolates the (hot) electrons in the plug from the (colder) electrons in the central cell, so power must be used to heat only the former. If a thermal barrier is established, the plug electron temperature can be kept at a higher value than the central cell temperature and high electrostatic potential can be achieved in the plug to confine the ions. In order to 
establish the barrier, the ions are removed by the thermal barrier region by charge exchange with a neutral beam injected almost parallel to $\mathbf{B}$ : the negative charge difference creates the hump in the electrostatic potential needed for the barrier.

The second scheme is more practical, since it puts less stringent conditions on the engineering parameters (magnetic field and injected ion energy) of the two end plugs.

It is apparent that in order to maintain this configuration, external power must be injected in the two end cells. On the other hand, if the volume of the end cells is sufficiently smaller than the volume of the central cell, the contribution to the global energy balance of the end cells is negligible, and large values of $\mathrm{Q}$ can be achieved.

Detailed calculations of the ion confinement in the central cell show that the $n \tau$ value can be enhanced by a significant factor by the plugging potential. Typical estimates yield enhancement factors roughly given by

$$
e \phi / T_{i} \exp \left(e \phi / T_{i}\right)
$$

This has been experimentally shown in the first generation of TM experiments (as e.g. TMX at Livermore, see Post 1987 for details) where enhancements by an order of magnitude in ion and electron confinement was achieved. However, it is already apparent from Fig. 3.4 that to maintain the desired shape of the electrostatic potential requires very sophisticated tools that must work in all the relevant range of parameters and especially at high density (to achieve high fusion power).

An important aspect of the TM is its stability against flute-like interchange mode (i.e. pressure-driven modes with very little variation along the magnetic field line in order to minimize any stabilizing line-bending effect). The configuration is stable even in the absence of additional multipolar field in the central cell, due to the connection between the plasma in the central cell and the plasma in the end cells (typically made by baseball or Ying-Yang coils and therefore MHD stable). A second class of MHD modes are the ballooning modes: these modes are localized in the regions of unfavorable magnetic field line curvature inside the central cell (and therefore do not experience the stabilizing influence of the end cell) but produce a substantial line bending of the magnetic field lines. Calculations of ballooning mode stability show achievable beta values typically 50\% lower than those obtained for flute-like modes (see Post 1987 and references therein). However, the inclusion of kinetic effects, such as finite particle orbit width, can significantly increase the stability threshold (note that in the old 2XIIB experiment, beta values larger than 200\% were achieved in regimes with large particle orbits). In summary, beta values above $20 \%$ might be achievable by tandem mirrors.

The possibility of MHD-stable central cell without the superposition of a multipolar field has the important consequence of reducing radial transport. In conventional mirrors radial transport is negligible with respect to axial transport, but becomes important in tandem mirrors due to the improvement of axial transport by the plugging potential.

Note that there is an important difference between radial transport in mirrors and in toroidal systems. In the latter case radial transport is forced to be ambipolar: any mechanism that enhances the loss rate of one species produces a situation where the loss 
rate of the other species is also enhanced. This is not the case in mirrors: since there are two loss channels (axial and radial losses), radial ion losses can be balanced, for istance, by axial electron losses, without the need of increasing the cross field electron diffusion. This observation is the basis for the control of the radial transport through the end-plate potential: radial transport is influenced by the radial electric field; the electron lost by axial transport are collected on a plate that tends to become charged negatively, driving also the plasma to a negative potential; by inserting a variable resistance between the end plate and the wall, it is possible to act on the potential difference between the plasma and the wall, reducing the radial electric field and the radial transport.

After the first generation of TM experiments (TMX and GAMMA-6 at Tsukuba), key achievements include the following:

- the GAMMA-10 device at Tsukuba (still in operation), has a axisymmetric central cell (in order to minimize the radial transport), stabilized by quadrupolar magnetic wells coupled to it by "axisymmetrizer" transition coils (Fig.3.5). Outside of these "anchor" cells are axisymmetric mirror cells where the thermal barrier and the plugging potential are generated (e.g. see Cho (2004)). It has an axial length of 27 $\mathrm{m}$, and the total volume of the vacuum vessel is $150 \mathrm{~m}^{3}$. The central cell has a length of $6 \mathrm{~m}$ and a fixed limiter with a diameter of $0.36 \mathrm{~m}$; the magnetic-field intensity $\mathrm{B}_{\mathrm{m}}$ at the midplane is $0.405 \mathrm{~T}$ with a mirror ratio $\mathrm{R}$ of 5.2. Ion-cyclotron heating (ICH) $(200 \mathrm{~kW}$ at 4.47 or $6.36 \mathrm{MHz}$, as well as $100 \mathrm{~kW}$ at 9.9 or 10.3 $\mathrm{MHz}$ ) are employed for the central-cell hot-ion production and the anchor stabilization, respectively. The axisymmetric end cells have an axial length of 2.5 $\mathrm{m}\left(\mathrm{B}_{\mathrm{m}}=0.497 \mathrm{~T}\right.$, and $\left.\mathrm{R}_{\mathrm{m}}=6.2\right)$.

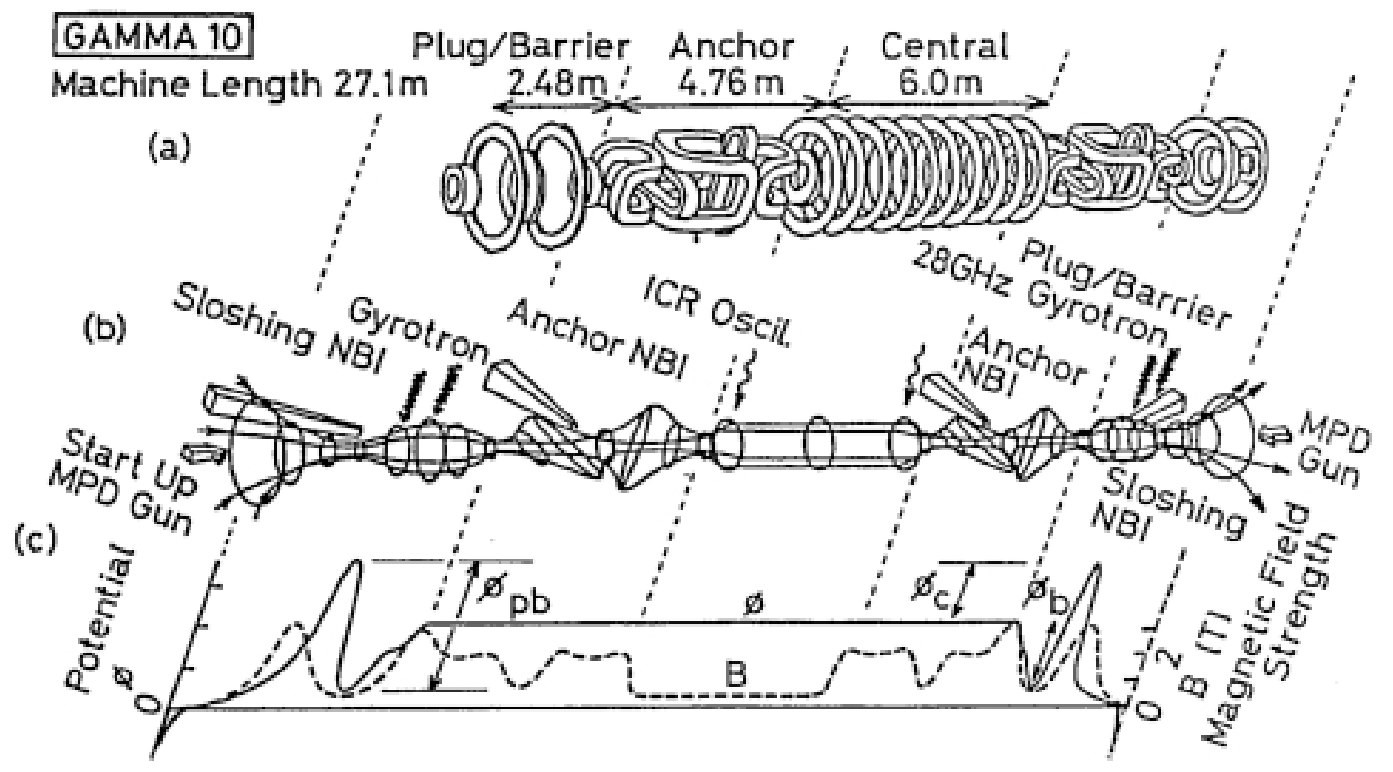

Fig.3.5 Schematic view of the GAMMA 10 tandem-mirror (from Cho (2004); (a) magnetic coil set, (b) magnetic-flux tube with heating systems, as well as (c) axial magnetic-field (dashed curve) and potential profiles (solid curve). 
- the Tandem Mirror EXperiment (TMX-U) at Livermore (e.g. see Simonen (1989)) had quadrupolar mirrors at the end of the central cell; these were connected to quadrupolar (MHD-stable) magnetic wells where thermal barrier and plugging potential were generated. Before being shut-down in 1988, TMX-U was able to demonstrate the thermal barrier concept at modest densities $\left(1-3 \times 10^{18} \mathrm{~m}^{-3}\right)$; the theoretical design limit $\left(10^{19} \mathrm{~m}^{-3}\right)$ was not achieved (due to the lack of heating power, according to the interpretation of the Livermore team). The experiment has also confirmed the theoretical expectations about the stabilizing effects of a population of "sloshing ions" produced by oblique injection of neutral beams.

- The TARA experiment at MIT, aimed at testing the possible use of axisymmetric central and plug cells (in order to reduce radial transport) with MHD stabilization provided by two quadrupolar anchor cell located at each end, outside the region were plugging occurs.

Activities are being carried out at present also on the AMBAL M device at the Budker Institute in Novosibirsk and on the HANBIT device in Korea. The large MFTF-B tandem mirror facility at Livermore was mothballed right after the test of the various systems in 1986, due to budgetary constraints.

All the experiments above have successfully demonstrated the validity of the TM concept (both the conventional and the thermal barrier scheme), and, in particular:

- the effectiveness of the electrostatic plug in suppressing ion end losses (with axial confinement time up to $0.7 \mathrm{~s}$ achieved in GAMMA-10); detailed measurements performed in TMX-U show very good agreement between the experimentally measured electrostatic potential and the theoretical predictions;

- the generation of thermal barriers at low density; unfortunately maintaining a steady-state thermal barrier at high density has not yet been proven;

- the ability to control radial transport by controlling the radial electric field in the central cell; radial ion confinement times above 1s have been achieved in GAMMA-10 (with an axisymmetric central cell) and about 0.1s in TMX-U (with non-axisymmetric central cell);

- the effectiveness of the ambipolar potential to isolate the electrons from thermal contact with the outside region, reducing the effective electron parallel thermal conductivity, with the electron temperature achieving values in the range of $300 \mathrm{eV}$;

- the ability of maintaining MHD stability by using minimum-B anchor cells;

- the possibility of suppressing high-frequency micro-instabilities due to sloshing ions and trapped warm plasma.

This said, the extrapolation of these results to plasma an order of magnitude larger in density and potential is still a question. When compared with fusion reactors (and taking the central cell parameters), the present results still need a substantial extrapolation both in $\rho_{*}$ (by about a factor 10) and in $\beta$ (a factor 5), whereas the values of the collisionality parameter $\nu_{*}$ would be similar to those obtained in present devices. 


\subsubsection{Field Reversed Mirror.}

In a Field Reversed Mirror (Fig.3.6) the plasma confinement is achieved by producing a ring current of energetic particles (typically by external neutral beam injection). If the current in the ring is sufficiently large, field reversal occurs and a napkin-ring shaped configuration is produced with closed magnetic field lines which confine the plasma. This concept was pioneered by the ASTRON (Gormezano 1979) device where field reversal was attempted with a beam of particles characterized by orbit size comparable with the device dimensions.

The Field Reversed Mirror has very much in common with "compact tori" configurations and therefore will be discussed later.

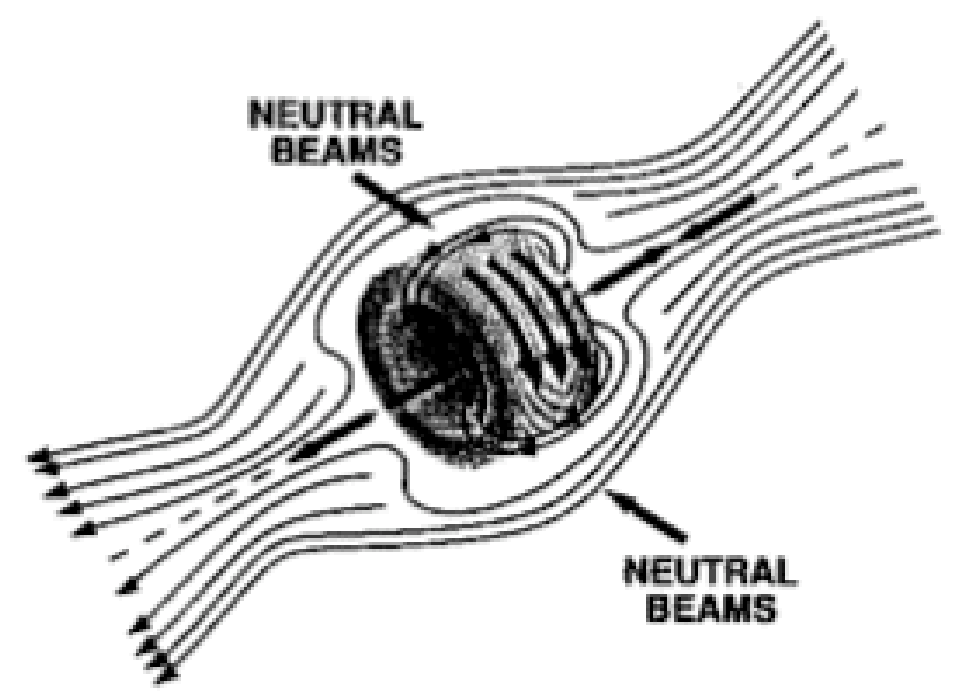

Fig.3.6 Field Reversed Mirror

\subsubsection{Gasdynamic Mirror.}

A Gasdynamic Mirror (Mirnov et al. (1979), Fig.3.7) is a mirror configuration characterized by a mean free path shorter than the longitudinal dimension $\mathrm{L}$ of the configuration and by a high mirror ratio $(\mathrm{R}>10)$. Due to frequent collisions, the plasma confined in the trap is very close to an isotropic Maxwellian state, and, therefore, many instabilities, potentially dangerous for classical magnetic mirrors with a collisionless plasma, generally cannot be excited. Moreover, in contrast with conventional mirrors, longitudinal plasma losses are insensitive to the ion angular scattering rate that might be enhanced by microinstabilities. Increased stability properties, obtained by minimizing the curvature of magnetic field lines driving plasma instabilities, enable large $\beta$. In a gasdynamic mirror the confinement time scales as

$$
\tau \approx \mathrm{LR} / \mathrm{v}_{\mathrm{ti}}
$$

(with $\mathrm{v}_{\mathrm{ti}}$ the ion thermal velocity) which shows a much stronger dependence on the mirror ratio than a conventional mirror. Furthermore, the confinement time depends on the system size, unlike ordinary mirrors. 

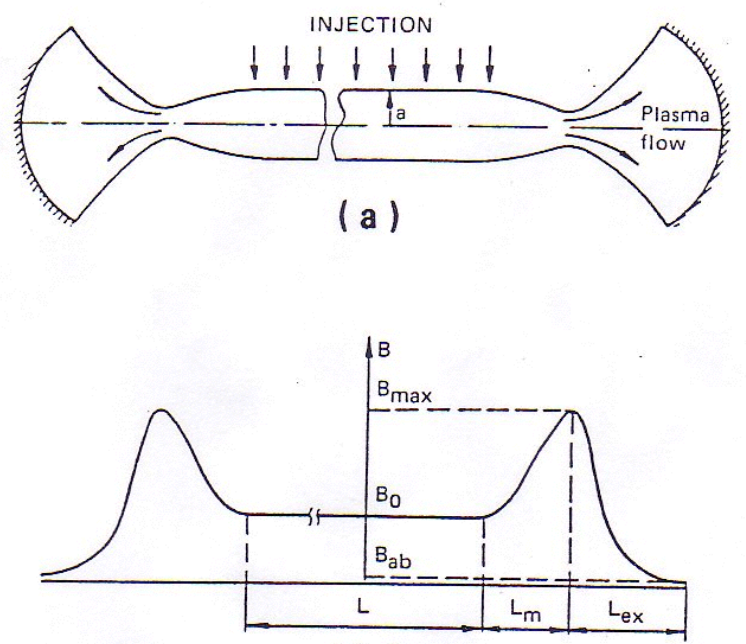

(b)

Fig. 3.7 Layout of a gasdynamic mirror from Nagornyj (1984): a) magnetic field lines; b) magnetic field strength on the axis. $B_{\max }, B_{0}$, and $B_{a b}$ stand for the magnetic field value in the mirror, the solenoid and the absorber; $\mathrm{L}, \mathrm{L}_{\mathrm{m}}$ and $\mathrm{L}_{\mathrm{ex}}$ are the lengths of the solenoid, of the mirror and of the expander, respectively; $a$ is the plasma radius in the solenoid

The short mean free path condition can be expressed as

$$
\mathrm{v}_{\mathrm{ti}} \tau_{\mathrm{ii}}<<\mathrm{LR}
$$

(note the extra factor R). Therefore, short mean free path and high confinement requires long configurations and large mirror ratio. It can indeed be shown that for energy production using the DT reaction, the mirror length should be in the range of $10 \mathrm{~km}$ at plasma densities around $10^{21} \mathrm{~m}^{-3}$ and mirror ratio $\mathrm{R}=50$. Since the Lawson parameter is proportional to $\mathrm{nL}$, shorter configurations can be achieved by higher density values. With a plasma radius of $0.1 \mathrm{~m}$, such a device would produce fusion power in the range of a few tens of GW. The neutron power density would be around $10 \mathrm{MW} / \mathrm{m}^{2}$. Higher values of the plasma density would reduce the size of the configuration but would also increase the neutron wall load above the limit presently considered achievable.

Presently, the only gasdynamic mirror is in operation at the Budker Institute in Novosibirsk (Kruglyakov (2002)). It consists in a device with a mirror-to-mirror distance of $7 \mathrm{~m}$, magnetic field up to $0.3 \mathrm{~T}$ in the midplane and up to $15 \mathrm{~T}$ at the mirror and a radius at the midplane of $8-15 \mathrm{~cm}$. Oblique neutral beam injection at $15 \mathrm{keV}$ is used for plasma heating up to a power level of $4 \mathrm{MW}$. The fast ions are reflected inside the mirror and density peaks in the outer part of the central cell, where fast ion densities up to $10^{19} \mathrm{~m}^{-3}$ have been produced. Target plasma density values in the range $3-20 \times 10^{19} \mathrm{~m}^{-3}$ have been produced with electron temperature values up to $130 \mathrm{eV}$. 
On this device it has been demonstrated that MHD plasma stability can be achieved in axially symmetric magnetic fields. Flute modes were stabilized by using external axisymmetric anchor cells in which the field line curvature was favorable for stability. As a result, on axis $\beta$ values exceeding $40 \%$ were almost entirely associated with the fast ion population.

The gasdynamic mirror has been also proposed as a possible volumetric neutron source. When compared with fusion reactors and taking the central cell parameters, the present results need to be extrapolated by about a factor 5 in $\rho_{*}$, and 2.5 in $\nu_{*}$, whereas the values of $\beta$ would within $50 \%$ those obtained in the present device.

A second gasdynamic mirror experiment has been just completed at the NASA Marshall Space Flight Center (Emrich 2002) to investigate the stability limits of this configuration.

\subsubsection{Other mirror concepts.}

Other mirror concepts have been proposed over the years such as the multiple mirrors (a configuration with many identical mirror cell linked together) and the rotating mirror (with the plasma rotating around the symmetry axis subject to a radial electric field that induces an ExB drift in the poloidal direction). These concepts, that are still in a preliminary stage of development but have an underlying physics on a large extente similar to the other mirror concepts, will not be further considered here.

At present, experiments on multiple mirrors are still carried out on GOL-3 at Novosibirsk (see e.g. Kruglyakov (2002)).

\subsubsection{Mirror studies for space propulsion}

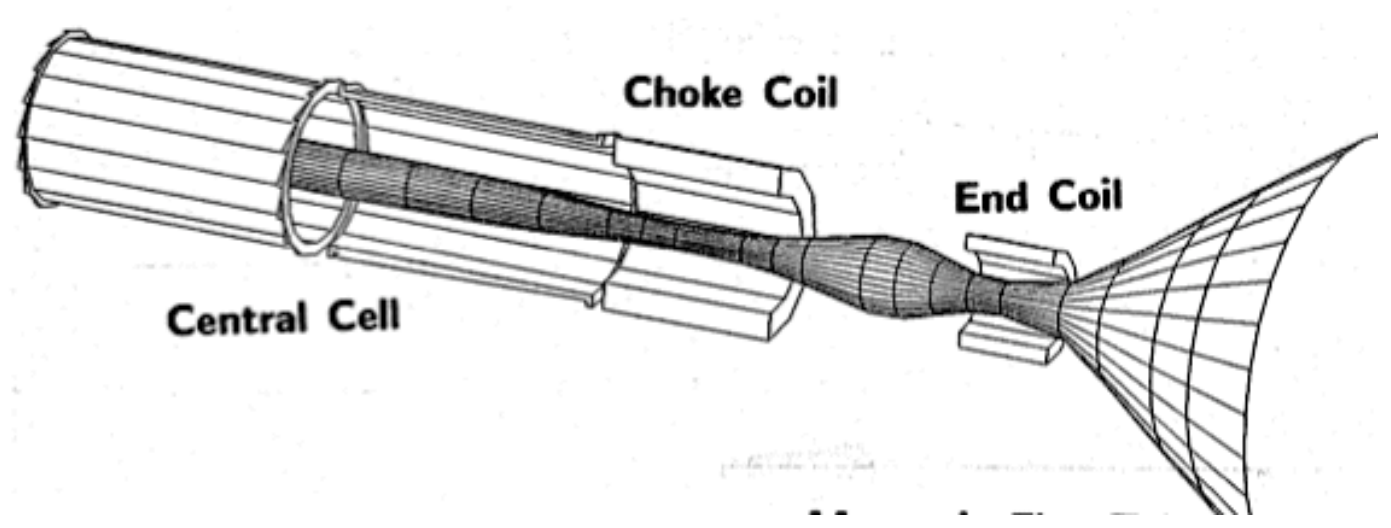

Fig. 3.8 SOAR

Several studies have involved the tandem mirror as a space propulsion system. Here we consider the study presented in Kulcinski (1987) for a Space Orbiting Advanced Fusion Reactor (SOAR), see Fig.3.8. Although the system was proposed for energy production only, its features are similar to those of a propulsion system. The electric power is $1 \mathrm{GW}$ and is produced by the $\mathrm{D}^{3} \mathrm{He}$ reaction through direct conversion ( $80 \%$ efficiency). The 
fusion power is $1.9 \mathrm{GW}$ with $70 \mathrm{MW}$ in the form of neutrons; about $470 \mathrm{MW}$ are lost through radiation. The shield is designed to absorb all the rejected heat, without using a radiator. An optimized $\mathrm{LiH}$ is employed with a total mass of about 300t. The length of the central cell is $73 \mathrm{~m}$ with a radius of $0.55 \mathrm{~m}$. The total mass of the system is $500 \mathrm{t}$, with an equivalent specific power of $2 \mathrm{~kW} / \mathrm{kg}$. The magnet system is axisymmetric and uses superconducting coils with 7.7T (NbTi) for the central cell, 18T (Nb3Sn) choke coils and $12 \mathrm{~T}$ end coils. The auxiliary heating power is $70 \mathrm{MW}$ and is produced with $75 \%$ efficiency.

The possibility of using a gasdynamic mirror for space propulsion has been considered by Kammash (1995b). The reactor has a central cell 50m long with a radius of $7 \mathrm{~cm}$ and a magnetic field of $15 \mathrm{~T}$. A high density $\left(\approx 5 \times 10^{22} \mathrm{~m}^{-3}\right)$, low temperature $(\mathrm{T} \approx 6.5 \mathrm{keV})$ DT plasma is sustained by the injection of $40 \mathrm{GW}$ of neutral beams with an injection energy of $20 \mathrm{keV}$. The energy confinement time is about $3 \mathrm{~ms}$. The fusion gain is only $\mathrm{Q}=1$. The high plasma density produce a very high neutron wall load $\left(\approx 622 \mathrm{MW} / \mathrm{m}^{2}\right)$, well above the values considered for terrestrial fusion power generation plant $\left(<5 \mathrm{MW} / \mathrm{m}^{2}\right)$. Taking the already mentioned fluence limit of $15 \mathrm{MWyr} / \mathrm{m} 2$, such a neutron wall load would limit the duration of full power operation to about 9days. No specific layout is provided. The thrust power is $55 \mathrm{GW}$. The rest of the power $(\approx 36 \mathrm{GW})$ must be radiated in space and this is the reason for the large radiator mass $(\approx 7200 t)$, which is the dominant component. For a radiator capable of radiating $5 \mathrm{~kW}$ per each $\mathrm{kg}$ of mass, the resulting specific power is in the range $7 \mathrm{~kW} / \mathrm{kg}$. The possibility of using $\mathrm{D}^{3} \mathrm{He}$ has also been considered in the same study. With the same dimensions and the same gain factor, the magnetic field must be increased up to $185 \mathrm{~T}$, the injection energy up to $200 \mathrm{keV}$ and the fusion power to $147 \mathrm{GW}$. The increase in the volume also increases the radiator mass $(>300000 t$ !) but the specific power is also increased $(\approx 80 \mathrm{~kW} / \mathrm{kg})$. How the electricity is produced for the neutral beam power supply is not discussed. All these figures and especially those associated to the energy budget are somewhat inconsistent and should be looked at with the benefit of doubt. 


\subsection{Field Reversed Configurations}

Compact toroids are configurations characterized by no mechanical structure linking the plasma. The configuration is "compact", in the sense that plasma extends to the geometric axis, and "toroidal", in the sense that the topology of the closed magnetic surfaces is that of a torus (Fig.3.9). Compact toroids can therefore combine the good confinement properties of closed toroidal configurations with the simple topology of open magnetic field systems.

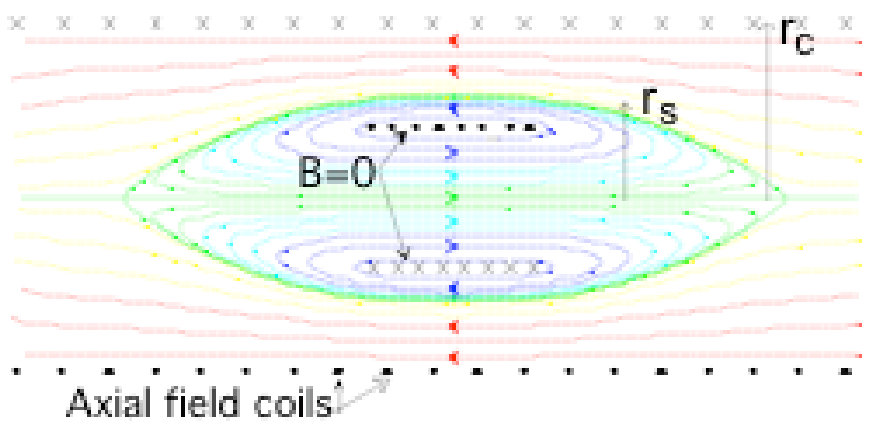

Fig.3.9 Field reversed configuration

Compact Toroids consist of two distinct regions:

- a closed field line region inside a magnetic separatrix, with radius $r_{s}$;

- an open field line sheath outside the separatrix.

Plasma is well confined inside the separatrix and exhausted through the open field line region.

Compact Toroids can be classified according to the following two parameters:

- the ratio between the poloidal magnetic field $\mathrm{B}$ (in the $(\mathrm{r}, \mathrm{z})$ plane) and the toroidal magnetic field $B_{\theta}$ (along $\theta$ );

- the parameter (usually denoted with S) corresponding to the number of ion gyroradii between the field null and the separatix. This parameter is related to the inverse of the $\rho_{*}$ parameter.

\begin{tabular}{|l|l|l|}
\hline & S $>1$ & S $<1$ \\
\hline $\mathrm{B}>>\mathrm{B}_{\theta}$ & $\begin{array}{l}\text { Field Reversed Configuration (FRC) } \\
\text { Field Reversed Mirror (FRM) }\end{array}$ & \multirow{1}{*}{ ASTRON } \\
\cline { 1 - 2 }$\approx \mathrm{B} \approx \mathrm{B}_{\theta}$ & $\begin{array}{l}\text { Spheromak } \\
\text { Field Reversed Mirror (FRM) }\end{array}$ & \\
&
\end{tabular}

In this section Field Reversed Configurations (FRC) are described; the next section is devoted to spheromaks.

3.3.1 FRC formation and equilibrium

The FRC is a variety of compact toroid with the following characteristics: no appreciable toroidal field, values of beta of order unity, no rotational transform, all the equilibrium 
current maintained by diamagnetism, a scrape-off layer exhausting heat and particles outside the coil system. FRC are reviewed in the paper by Tuszewski (1988).

FRCs were accidentally discovered in $\theta$-pinches in the ' $60 \mathrm{~s}$. In order to understand the main features of this configuration it is useful to consider the main formation scheme (the $\theta$-pinch formation) illustrated in Fig.3.10:

a. the discharge tube is filled with neutral gas and a bias magnetic field is applied; the gas is pre-ionized freezing the magnetic field in the plasma with a temperature of a few eV;

b. the current in the theta pinch coils is reversed on a fast time scale, inducing in this way a plasma current along $\theta$ (and an axial field opposite to the bias field) that causes the plasma and bias field to implode radially;

c. the oppositely directed magnetic field lines reconnect near the end of the thetapinch coil, forming a closed magnetic field configuration;

d. the large magnetic tension at the reconnection region causes the FRC to contract in the axial direction until an equilibrium configuration is achieved.

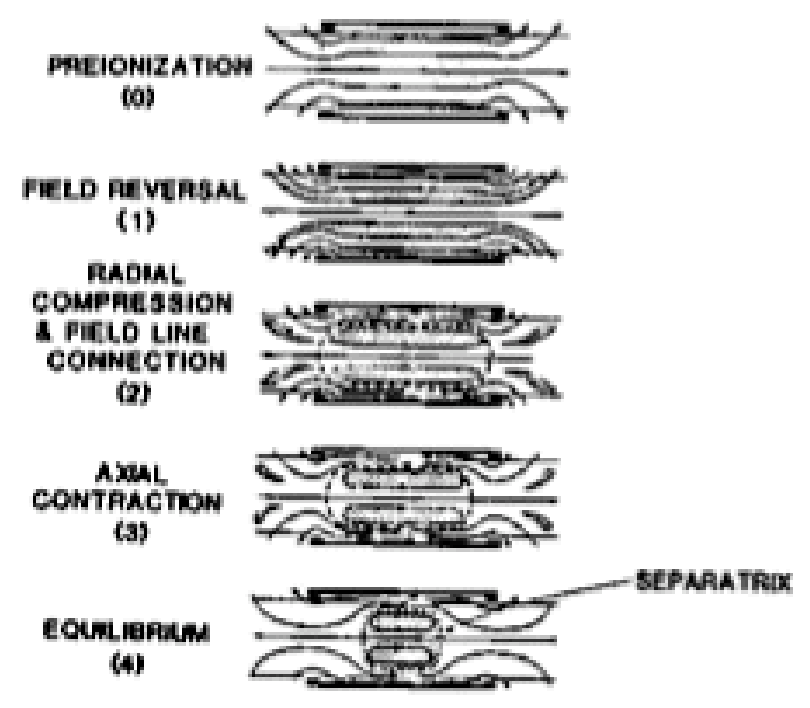

Fig.3.10 FRC formation sequence

During phase $b$ heating occurs with a shock followed by slow compression; resistive heating also occurs during the annihilation of the bias field and is characterized by a resistive dissipation higher than classical.

The main interest of FRCs is due to the fact that in order to achieve an equilibrium configuration the average plasma beta must be high. Using simple analytical models (confirmed by fully numerical analysis), it can be shown that

$$
<\beta>=1-\mathrm{r}_{\mathrm{s}}^{2} / 2 \mathrm{r}_{\mathrm{c}}^{2},
$$


with $r_{s}$ and $r_{c}$ being the separatrix radius and the flux conserver radius, respectively. Since $r_{s} \leq r_{c}$, this implies beta values larger than 50\%. Nevertheless the plasma maintains remarkable stability properties.

The flux $\phi$ of the axial magnetic field between the null point and the separatrix can be shown to be bound by two values:

$$
\phi \equiv \int_{\mathrm{R}}^{\mathrm{r}_{\mathrm{s}}} \mathrm{B} 2 \pi \mathrm{rdr}=\pi \mathrm{r}_{\mathrm{c}}^{2} \mathrm{~B}_{\mathrm{e}}\left(\mathrm{r}_{\mathrm{s}}^{2} / 2 \mathrm{r}_{\mathrm{c}}^{2}\right)^{(3+\mathrm{k}) / 2}
$$

with $\mathrm{B}_{\mathrm{e}}$ being the magnetic field outside the separatrix (determined by the poloidal coil current) and the two boundary values obtained for $\mathrm{k}=0$ and $\mathrm{k}=1$, respectively. From the above expression it is possible also to determine an expression for the parameter $\mathrm{S}$

$$
\mathrm{S}=\phi /\left(2 \pi \mathrm{r}_{\mathrm{s}} \rho_{\mathrm{ie}} \mathrm{B}_{\mathrm{e}}\right)=2^{-3 / 2}\left(\mathrm{r}_{\mathrm{c}} / \rho_{\mathrm{ie}}\right)\left(\mathrm{r}_{\mathrm{s}}^{2} / 2 \mathrm{r}_{\mathrm{c}}^{2}\right)^{(2+\mathrm{k}) / 2}
$$

with $\rho_{\text {ie }}$ being the ion gyroradius in the external magnetic field. Therefore the parameter $S$ is always lower than the value obtained for $r_{s}=r_{c}(\beta=50 \%)$ and $k=0$, i.e. $S<r_{c} / 5 \rho_{\text {ie }}$.

3.3.2 Open issues in FRC research

The main issues of FRC, briefly discussed below, can be grouped in stability, formation, sustainment, transport and technology development. For more details see Steinhauer (1998)

Stability. FRCs are high beta configurations and might be expected to be MHD unstable. Indeed a FRC is the toroidal version of the Z-pinch which is well known to be unstable for sausage and kink modes in the absence of a longitudinal (toroidal in the case of FRC) magnetic field. Contrary to these expectations, current FRC experiments are not limited by instabilities. Specifically:

- Ideal MHD modes. The most serious instability predicted in FRC is the internal tilt mode which breaks the toroidal flux surfaces and corresponds to the kink mode in a Z-pinch (for small plasma elongation also the external tilt mode, which produces a flip in the plasma axis, may be unstable). No observation of the internal mode has been reported so far.

- Tearing modes. Tearing modes are observed during the formation phase but the subsequent equilibria appear to be stable to tearing modes.

- Rotational modes. Following the formation phase, the plasma start to rotate in the ion diamagnetic direction. Although the origin of plasma rotation is not fully understood, it is clear that rotation causes new instabilities. The most dangerous is the $n=2$ rotational instability that can destroy the configuration. A threshold in the ratio $\alpha \equiv \Omega / \Omega_{\mathrm{Di}}$ (with $\Omega$ the rotation frequency and $\Omega_{\mathrm{Di}}$ the ion diamagnetic rotation frequency) in the range $\alpha \approx 1.5$ is predicted by theory. The mode is suppressed by applying a multipolar field by external coils with straight or helical windings.

The fact that many instabilities predicted are not actually observed in FRCs is not surprising. Several effects can play a stabilizing role: 
- The parameter S (the number of ion gyro-radii between the field null and the separatix) is of the order $S \approx 1-2$ in current experiments. In these conditions several kinetic effects can play a stabilizing role: orbit width comparable with the perpendicular mode wavelength, diamagnetic frequency comparable with the Alfvén growth rate, finite plasma compressibility. Note also that the MHD model is not adequate in this limit. Thus, the most important question is whether FRC will remain stable also in reactor-relevant conditions with projected values of $S$ in the range $S=30-40$.

- The low-beta open field region is MHD stable because of the favorable curvature of the magnetic field line at the end of the configuration. This effect can help in stabilizing the FRC core.

- The presence of a conducting boundary and of toroidal rotation can play a stabilizing role.

There is not yet quantitative agreement between experimental results on FRC stability and theoretical analyses, although the role of kinetic effects is widely recognized. Thus, an extrapolation to a next generation of FRC experiments is not yet possible. Since it is clear that the requirement of larger $\mathrm{S}$ values (for better confinement) is conflicting with the requirement of bulk plasma stability, additional stabilizing mechanisms should be investigated. For example, it has been suggested to produce an energetic ion-ring, by the injection of energetic ions carrying most of the equilibrium current, which at the same time would provide both a stabilizing mechanism and a mean to sustain the configuration in steady state. This approach has been already used in the ASTRON device and in the Field Reveresed Mirror experiments and it is currently proposed for the Colliding Beam Fusion Reactor (CBFR) discussed below (Rostoker 1993).

Formation. The theta-pinch formation sequence produces FRC on a time scale of a few Alfvén times and it would imply large amount of pulsed power when extrapolated to a reactor. Slow FRC formation schemes aims at formation over the resistive time scale (a few order of magnitude longer) by the Coaxial Slow Source, the Rotamak, the Extrap and the Field Reversed Mirror. These methods are described in Tuszewski, (1988). Their extrapolability to reactor conditions must be proved.

An interesting feature of FRCs, related to their stability, is the possibility of translating the configuration along the symmetry axis away from the formation region through a weak gradient in the axial field. This property allows a better adiabatic compression heating and a physical separation of the high technology formation chamber from the burn and quench chambers. This possibility is particularly interesting in the context of the so called Magnetized Target Fusion approach (Siemon 1999): the FRC is translated inside a metallic liner which is then imploded on a microsecond time scale (Taccetti 2003). This approach is intermediate between the magnetic and inertial confinement schemes and is illustrated in Fig. 3.11. 


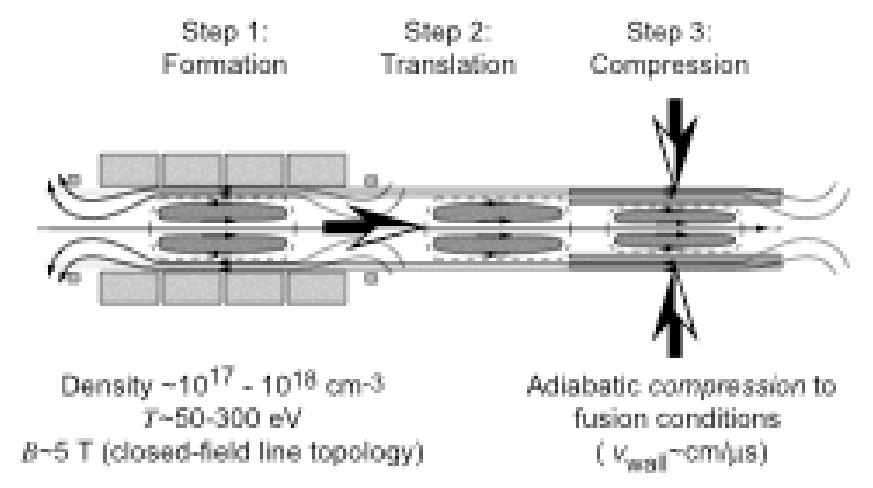

Fig.3.11 The three steps of FRC- based MTF approach (from Taccetti (2003)).

Sustainment. In present experiments, the life time of the configuration depends on the rate at which the magnetic flux $\phi$ initially trapped, is dissipated. In order to maintain the configuration in steady-state conditions several methods have been proposed and need to be tested: rotating magnetic fields (tested only in cold plasmas), neutral beam current drive and spheromak merging.

- In the case of rotating magnetic field (RMF) current drive a small rotating transverse field component is generated by oscillating currents driven in longitudinal conductors located near the wall. Under certain frequency and collisionality conditions, the transverse field penetrates the plasma and drives an electron current in a manner similar to an induction motor. This method has been proven but only in cold devices called rotamaks. Experiments are ongoing to demonstrate its applicability to hotter plasmas.

- Neutral beam injection experiments could sustain the configuration for times much longer than $1 \mathrm{~ms}$. Injection of $100 \mathrm{~A} 30-60 \mathrm{kV}$ beams would also induce a rotation with velocities in the order of the Alfvén velocity. As already noted, beam particles could also play a stabilizing role. This approach, also used in the CBFR, has been used in Field Reversed Mirrors.

- Spheromak merging has been shown on TS3 to produce a FRC configuration if the two spheromaks have opposite helicity.

Transport. Turbulent transport has been observed also in FRC. Turbulence affects not only the cross-field particle and energy transport, as in tokamaks, but also the decay of the poloidal magnetic flux (anomalous resistivity). In the scrape-off layer anomalously slow particle outflow has been also detected.

Some understanding exists only for the cross field particle/energy transport which is consistent with the expectations of low-frequency drift-wave turbulence. Several small scale instabilities have been considered: the lower-hybrid drift instability, the microtearing modes driven by the electron temperature gradient and the KelvinHelmholtz instability driven by velocity shear. Classical losses associated with unconfined particle in velocity space (as in the simple mirror configuration) in the region close to the separatrix have been also proposed. The present diagnostic capabilities allow to determine with reasonable accuracy the particle confinement time $\tau_{\mathrm{N}}$. The particle 
losses appear to account for $60-80 \%$ of the energy losses, the remaining part being associated with radiation and thermal conduction. The measured values of $\tau_{\mathrm{N}}$ is in the range $10-200 \mu$ s and scales linearly with the parameter $\mathrm{R}^{2} / \rho_{\mathrm{ie}}$, as shown in Fig.3.12, with $r_{s}=2^{1 / 2} R$

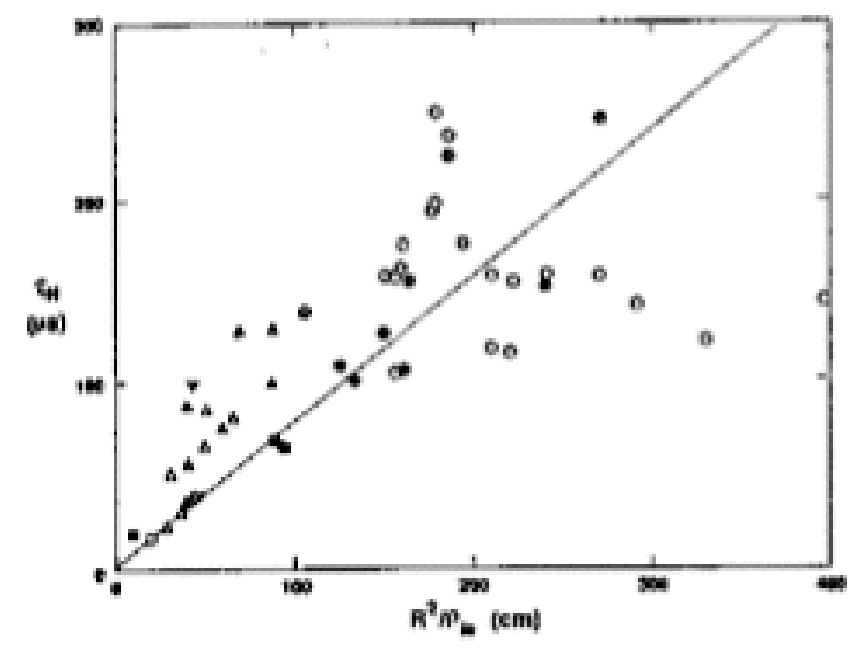

Fig.3.12 Scaling of the particle confinement time

This empirical scaling is more or less consistent with theoretically based scaling derived from quasi-linear estimates of the turbulent transport and clearly shows the apparently conflicting requirements of stability (low S) and good confinement (high S). Classical transport is not consistent with the observed trends, although the ratio between the experimental and the classical value of the confinement time can be as low as 3 . As to the characteristic decay time of the poloidal flux, the comparison between the experimental value and that derived from the classical Spitzer resistivity shows a discrepancy ranging between 2 and 20, showing the presence of substantial turbulence effects. Finally, we note that changes in the turbulence regimes (and therefore in global transport) may be expected for the FRC at larger values of $\mathrm{S}$.

Technology. A research program is presently being pursued in a joint U. Washington / LANL effort, to develop the best method for the generation of rotating magnetic fields including: (1) design and construction of a suitable high power RF source and drive coils capable of a sustained pulse longer than $1 \mathrm{msec}$; (2) demonstration of the RMF technique in a plasma column of moderate size (0.5-m diameter and $1.5-\mathrm{m}$ length); (3) investigation of alternate methods for generating the RMF which are more efficient and capable of delivering higher power.

\subsubsection{Present FRC experiments.}

Parameters achieved so far in the various FRC facilities range from $5 \times 10^{19}$ to $5 \times 10^{21} \mathrm{~m}^{-3}$ in plasma density, $3 \mathrm{keV}$ ion temperature and $0.5 \mathrm{keV}$ electron temperature, plasma beta in the range $0.75-0.95$. The high values of plasma density are particularly remarkable although obtained in the first generation, short duration experiments. As the configuration 
lifetime increases, the trend is towards lower density values. Typical values of $r_{s} / r_{c}$ are in the range 0.4-0.6, although values up to 0.9 have been achieved. Elongations in the range 3 to 10 have been obtained. Values of the Lawson parameter $n \tau$ as large as $10^{17} \mathrm{~m}^{-3} \mathrm{~s}$ have been obtained.

Research in FRC is carried out mainly in US, Russia, Japan. The main facilities are listed below:

$\mathrm{BN}$ (TRINITI research center, Troitsk, Russia). This facility $\left(1=0.9 \mathrm{~m}, \mathrm{r}_{\mathrm{c}}=0.21 \mathrm{~m}\right.$, $\mathrm{B}=0.45 \mathrm{~T}, \tau=50 \mu \mathrm{s}$ ) has investigated improved control techniques, internal magnetic field structure and electron energy distribution. Has been used also to form different magnetic configurations (spheromak and tokamak).

TL (TRINITI research center, Troitsk, Russia). This facility uses independent active endcontrol coils for dynamic formation and has investigated start-up methods with different time scales.

TOR (TRINITI research center, Troitsk, Russia). This facility $\left(1=1.5 \mathrm{~m}, \mathrm{r}_{\mathrm{c}}=0.3 \mathrm{~m}, \mathrm{~B}=1 \mathrm{~T}\right.$, $\tau=100 \mu \mathrm{s})$ has investigated the strong heating that occurs during the start-up.

NUCTE-3 (Nihon University, Japan). This facility ( $\left.1=2 \mathrm{~m}, \mathrm{r}_{\mathrm{c}}=0.16 \mathrm{~m}, \mathrm{~B}=1 \mathrm{~T}, \tau=60 \mu \mathrm{s}\right)$ has investigated the global modes dynamics and the control of the separatrix shape by auxiliary coils. The effect of a multipolar field on stability and confinement has been also investigated.

FIX (Osaka University, Japan). This facility generates FRCs (using a theta pinch source) that are then translated in a large chamber where they expand. The reduction of density following the expansion $\left(5 \times 10^{19} \mathrm{~m}^{-3}\right)$ enables the use of neutral beam injection (Okada 2004).

TS-3/TS-4 (Tokio University, Japan). The TS-3 facility has been employed for the formation of a variety of magnetic configurations (FRCs, spheromaks and ultra low aspect ratio tokamaks). FRC have been formed by counter.helicity merging of two spheromaks (the helicity concept is introduced in the next section). TS-3 has been recently upgradede in the TS-4 facility (Kawamori (2004)).

LSX/mod (University of Washington, USA). This is the largest FRC facility in the world $\left(\mathrm{l}=5 \mathrm{~m}, \mathrm{r}_{\mathrm{c}}=0.9 \mathrm{~m}, \mathrm{~B}=0.8 \mathrm{~T}\right)$. It has been converted in the TCS facility (with a confinement chamber at the end of the translation section) to perform experiments on the control the separatrix shape and on the start-up and sustainement by rotating magnetic fields (Hoffman 2004). This facility should provide information on the MHD stability at larger values of the parameter S (lower values of $\rho_{*}$ ).STX (University of Washington, USA). This facility $\left(1=3 \mathrm{~m}, \mathrm{r}_{\mathrm{c}}=0.4 \mathrm{~m}, \mathrm{~B}=0.2 \mathrm{~T}\right)$ is called the Star Thruster Experiment and is partially funded by NASA to investigate space relevant procedures for space propulsion such as rotating magnetic fields. Very powerful (but short lived) rotating magnetic fields will be used to overcome the ionization and radiation barrier that have so far limited the use of this technique to low temperature plasmas.

MRX/SPIRIT (Princeton, USA). The Magnetic Reconnection Experiment (MRX) can generate spheromaks, low aspect ratio tokamaks and FRCs. SPIRIT is a proposal to investigate MHD stability and confinement over a wide range of $\mathrm{S}$ (1-15) and elongation $\left(0.5<1 / 2 r_{s}<4\right)$. On a longer time frame, neutral beam injection could be used. 
FIREX (Cornell University, USA). The Field-reversed Ion Ring EXperiment injects an ion beam from a diode through a magnetic cusp to form an ion ring that should carry a large fraction of azimuthal current and provide stability.

ROTAMAK (Flinder University, Australia). In this facility, spherical FRCs have been produced and sustained up to $40 \mathrm{~ms}$ using up to $200 \mathrm{~kW}$ of rotating magnetic field power. The amount of driven current is presently limited by the available power.

FRX-L (Los Alamos) is a compact plasma injector to study high density FRC formation, stability, and translation physics, in preparation for its eventual use to demonstrate the physics of magnetized target fusion. Very high average densities (in the range $4 \times 10^{22} \mathrm{~m}^{-3}$ ) have been achieved with a (ion plus electron) temperature of $500 \mathrm{eV}$. Liner implosion tests have been carried out without plasma. The integrated plasma/liner experiments are scheduled for 2006 (Taccetti 2003).

\subsubsection{Use of FRC for space propulsion}

The use of FRC for space propulsion has been proposed first by Chapman (1989) using $\mathrm{D}^{3} \mathrm{He}$ fuel. Thrust is obtained by using a magnetic nozzle where plasma flowing along the open field line is mixed with propellant Fig.3.13. The design was largely based on the conceptual design of the land-based power plant SAFFIRE (Miley (1978)).

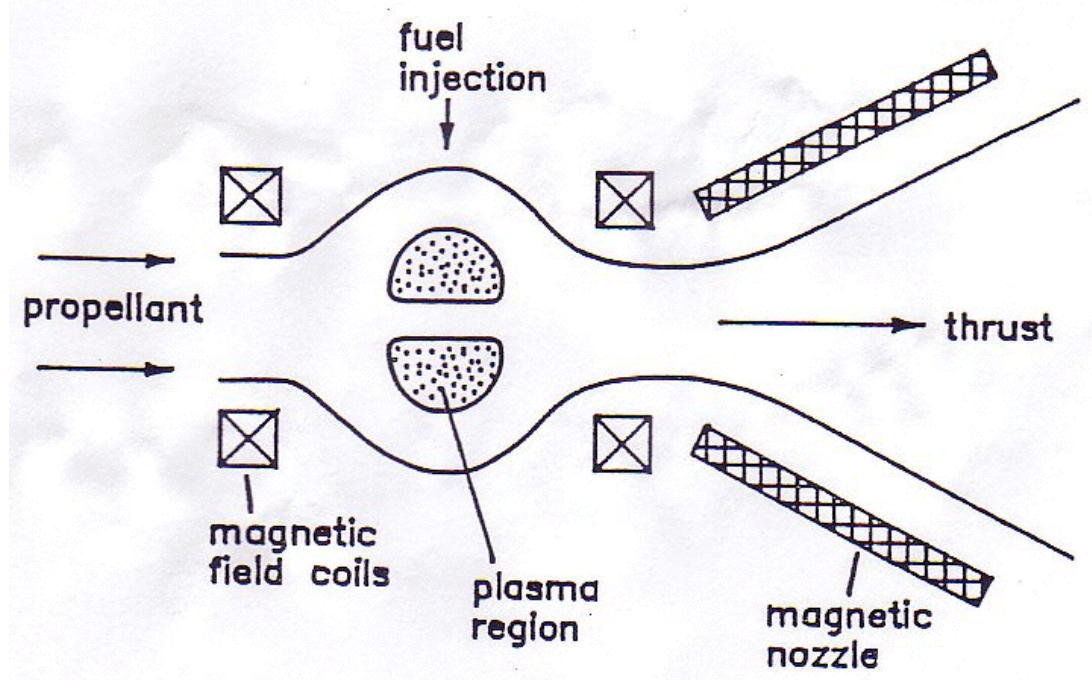

Fig.3.13 FRC propulsion concept from Chapman (1989)

The example considered in Chapman (1989) foresees the use of a $5 \mathrm{~T}$ magnet, $80 \mathrm{~m}^{3}$ plasma volume with a plasma radius of $1.5 \mathrm{~m}$. A confinement time of $2 \mathrm{~s}$ and a plasma beta of $76 \%$ are envisaged to produce fusion power at a level of $0.5 \mathrm{GW}$. Note that the parameter $\mathrm{S}$ for such a configuration would be around 50, well above the present values.

More recently, a colliding beam fusion reactor (CBFR) space propulsion system has been proposed (Cheung (2004)) shown in Fig.3.14 


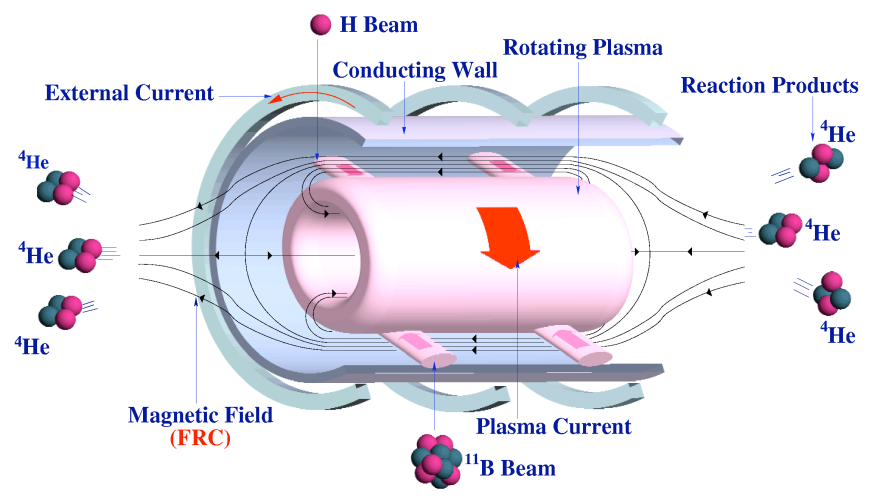

Fig.3.14 Colliding beam fusion reactor space propulsion system from Cheung (2004)

The reaction is the $\mathrm{p}^{-11} \mathrm{~B}$ (although also the DT and $\mathrm{D}^{3} \mathrm{He}$ reactions have been considered). The CBFR (Rostoker 2003) is an evolution of the ion ring concept mentioned above. Neutral beams are injected to produce a current that sustains the configuration. Electrons are confined by the radial electric field determined by the radial force balance of the fluid. Fusion products escape confinement and, to maintain charge neutrality, extract electron with sufficiently high energy to climb the electrostatic potential well. This results in a cooling of electrons and a reduction of Bremsstrahlung. The beams tend to thermalize, and this effect must be counteracted by continuous injection which requires a non negligible amount of re-circulating power (around $50 \%$ for the $\mathrm{p}^{-11} \mathrm{~B}$ case).

The CBFR for space propulsion has a chamber length is $6.9 \mathrm{~m}$ and the chamber radius $0.6 \mathrm{~m}$. The external magnetic field is about $0.5 \mathrm{~T}$. The CBFR generates about $77 \mathrm{MW}$ of fusion power $\left(\mathrm{P}_{\text {spec }} \approx 20 \mathrm{MW} / \mathrm{m}^{3}\right)$ and requires $50 \mathrm{MW}$ of injected power for steady-state operation. A direct energy converter intercepts approximately half of the alpha particles, decelerates them by an inverse cyclotron process and converts their energy into electricity. The remaining alpha particles are used for direct propulsion. The direct energy converter produces about $38.5 \mathrm{MW}$ of electricity. The remaining $11.5 \mathrm{MW}$ are produced from Bremsstrahlung by a thermoelectric converter (4.6MWe out of $23 \mathrm{MW}$ ). The part that is not converted is passed to a Brayton-cycle heat engine that supplies the remaining $7 \mathrm{MW}$. Waste heat (11MW) is rejected to space.

The mass distribution is shown in Table 1.1. The resulting specific power is about $3 \mathrm{~kW} / \mathrm{kg}$.

A propulsion system based on the Magnetized Target Fusion approach has been proposed (Thio 1999). A pair of conical theta-pinches produced a compact torus (either a FRC or a spheromak), that is imploded by a spherically converging plasma liner produced by a number of plasma jets. The liner is compressed to very high density with the production of an inner layer, made by the fusion fuel, which produces the main fusion yield, and an external layer, made by hydrogen, that slows down the neutrons, absorbing and converting $95 \%$ of their energy in charged particle energy. The spherically expanding 
plasma produced in this way is converted into an axial flow by a pulsed magnetic field. High conversion efficiencies into direct thrust are foreseen in the proposal.

The system is very compact. Higher radiator efficiencies (up to about $50 \mathrm{~kW} / \mathrm{kg}$ ) have been used in the study leading to a drastic reduction of the radiator mass. The reactor weight is only $41 \mathrm{t}$ for $25 \mathrm{MW}$ power production and the resulting value of specific power are astonishingly high $(400 \mathrm{~kW} / \mathrm{kg}$ which are reduced to about $100 \mathrm{~kW} / \mathrm{kg}$ if more conventional figures for the radiator mass are employed. The key to such a result is the use of the high fusion power density typical of the MTF approach and the conversion of the neutron power in charged particle power in the liner, which reduced the power to be radiated. Clearly, such a proposal is still at the conceptual stage and its feasibility can only be assessed after the experimental results of other Magnetize Target Fusion facilities such as FRX-L in the coming years. 


\subsection{Spheromaks}

A spheromak is a toroidal configuration in which no materials such as the vacuum vessel and the magnet link the torus. In this respect it is similar to FRC. Unlike FRC the strengths of the poloidal and toroidal fields are approximately equal. Spheromak research is reviewed in Jarboe (1994).

Spheromaks are relaxed configuration verifying the Taylor minimum energy principle (Taylor (1976)). According to such a principle, the magnetic configuration relaxes to a state which minimizes the energy $\mathrm{U} \equiv \int \mathrm{dV} \mathrm{B}^{2} /\left(2 \mu_{\mathrm{o}}\right)$ with the constraint of constant helicity $\mathrm{K} \equiv \int \mathrm{dV} \mathbf{A} \cdot \mathbf{B}$, with $\mathbf{A}$ the vector potential and $\mathbf{B}=\mathbf{\nabla} \times \mathbf{A}$ the magnetic field (the integral is over the plasma volume). The minimization of $\mathrm{U}$ with the constraint of $\mathrm{K}=$ constant, leads to the equation

$$
\nabla \times \mathbf{B}=\lambda \mathbf{B}
$$

with $\lambda$ a global constant. The solution of Eq.(3.10) is a force-free state $(\mathbf{J} \times \mathbf{B}=0)$. The minimum energy principle has been successfully applied to the description of the Reversed Field Pinch equilibrium, a plasma configuration which has several common features with Spheromaks. Note that, strictly speaking, relaxed states by definition have zero pressure gradient and are therefore irrelevant for plasma confinement. In practice, these configurations depart from a truly relaxed state and have finite pressure gradients.

\subsubsection{Spheromak formation}

Five different schemes are currently employed for spheromak formation: the flux core; the $\theta$-pinch z-pinch; the coaxial source; the conical $\theta$-pinch; the kinked z-pinch. These schemes are described in Jarboe (1994)). Only the coaxial source is reported here since it produces the best quality spheromaks (toroidal plasma current of 1MA, peak magnetic field of 3T, electron temperature of $400 \mathrm{eV}$, plasma density close to $10^{20} \mathrm{~m}^{-3}$ and energy confinement time of $0.2 \mathrm{~ms}$, for a pulse length of $10 \mathrm{~ms}$ ). For reference, the layout of the CTX experiment is shown in Fig.3.15. The formation sequence is shown in Fig. 3.16.

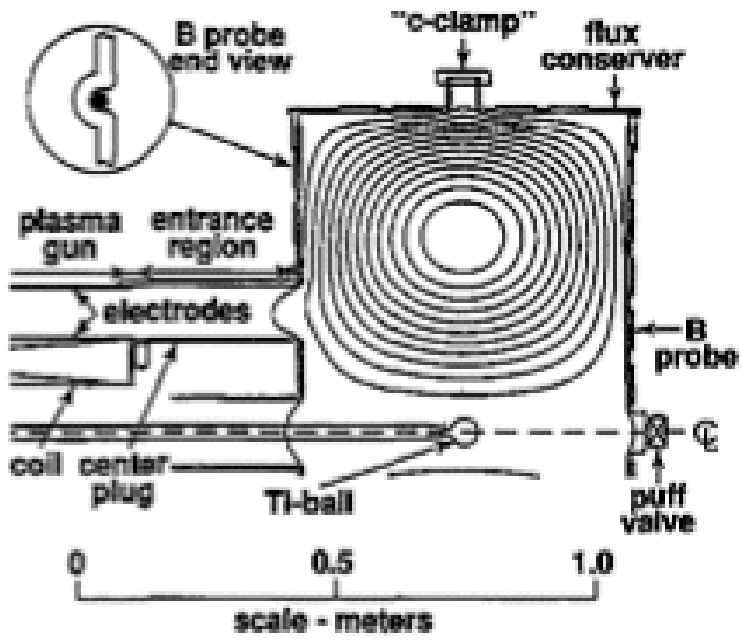

Fig. 3.15 Layout of the CTX experiment showing a formed spheromak (from Jarboe 1994) 

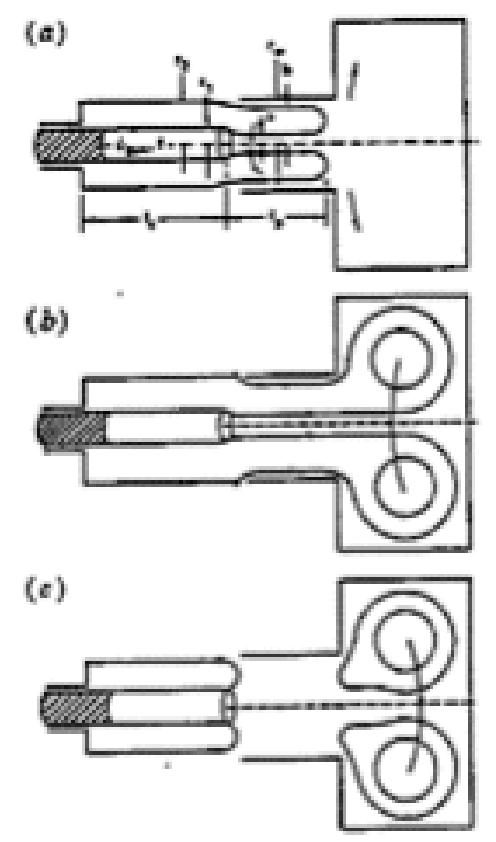

Fig.3.16 Spheromak formation sequence from Jarboe (1994)

The coaxial source is made of a pair of coaxial electrodes. Initially, a magnetic flux penetrates the inner electrode. Gas is injected between the electrodes and ionized to form a plasma which is frozen in the initial magnetic field. The electrode current is increased and, above a certain threshold, plasma and magnetic field are ejected from the source into the flux conserver. After the coaxial current drops below a threshold value the fields between the source and the spheromak reconnect and an isolated spheromak is formed. The coaxial source can be also used to sustain in steady-state conditions the spheromak configuration that otherwise would decay due to dissipation in the plasma. Note that the whole magnetic configuration, including the toroidal current in the plasmoid, is sustained, although the electric field produced by the gun is in the poloidal direction, orthogonal to the driven current. A similar situation arises in the reversed-field pinch system (Bodin and Newton 1980) where a poloidal current associated with field reversal is maintained by a toroidal electric field. The generation of magnetic field by the plasma is due to the so-called dynamo mechanism, which is typically a turbulent process. The drawback of this process is the generation of stochastic magnetic fields that can substantially reduce the confinement properties of these configurations.

\subsubsection{Stability limits}

The spheromak is generally considered a low-beta configuration. However, experimental values of beta in excess of $20 \%$ have been obtained, above the Mercier limit i.e. the beta limit for flute-like interchange modes (Jarboe 1994).

The most important unstable modes are briefly summarized below:

- Tilt mode. The dipole moment of a spheromak in a vertical field is anti-parallel to the magnetic field. Hence, in a uniform magnetic field the spheromak will tend to flip its axis to make the dipole moment parallel to the vertical field. The mode can 
be stabilized in a mirror field, but then the shift mode becomes unstable. If the equilibrium is provided by the flux conserver, instead that by a vertical field, the axisymmetric solution is stable for oblate flux conservers (i.e., for a cylindrical flux conserver, if the length of the cylinder is lower than 1.67 times the radius)

- Current driven modes. Current driven modes may become unstable when the $\mathrm{J}_{\|} / \mathrm{B}$ radial profile ( $\mathrm{J}_{\|}$is the current density component parallel to the equilibrium field) departs from a constant, which correspond to the minimum energy state predicted by theory $\left(\mathrm{J}_{\|}=\lambda \mathrm{B}\right.$ from Eq.3.10). Internal current driven modes have been observed in good agreement with the theoretical predictions.

- Pressure driven modes. The spheromak has unfavorable flux-surface averaged curvature everywhere. Ideal interchange instability can arise if the Mercier criterion is violated.

It should be noted that many spheromaks have been modified to insert a central conductor which makes the configuration evolve towards a (ultra) low aspect ratio tokamak. Such a modification is especially beneficial for the stabilization of the tilt mode (which is obviously opposed by the presence of a central conductor. Whether such a modified topology can still be of interest for space propulsion is a matter that should be further investigated.

\subsubsection{Confinement.}

Confinement in spheromaks is supposed to be heavily affected by the plasma turbulence that produces the dynamo effects, just as in reversed field pinches. The largest value of the energy confinement time $(\approx 0.2 \mathrm{~ms})$ has been obtained on CTX (Jarboe 1994). The local diffusivity is consistent with the expression, derived first by Rechester and Rosenbluth (1978), for the collisionless diffusion of a test particle in a stochastic magnetic fields .

It is not clear whether the poor energy confinement is an inherent feature. As noted above, relaxed configurations are sustained by the generation of magnetic fields through the dynamo process. Such a mechanism can produce stochastization of magnetic field lines and very poor confinement properties. The main issue is therefore to maintain the dynamo mechanism with the minimum amount of turbulence to keep energy confinement at an acceptable level. It should be noted that in the recent years encouraging results have been obtained in several reversed field pinch experiments where reduced transport by controlling the level of plasma turbulence has been achieved (Sarff 2002). For example, when the so-called Quasi Single Helicity states are produced (in which turbulence with a given helicity component dominates) the plasma volume with stochastic magnetic field lines is reduced and, as a consequence, confinement is improved.

\subsubsection{Present experiments}

The Sustained Spheromak Physics Experiment (SSPX) in operation at Livermore (see Wood (2004)) has the primary goal of testing whether a favorable energy confinement scaling can be obtained in a spheromak plasma sustained by coaxial helicity injection. Plasma temperature in the range of $200 \mathrm{eV}$ are reported, at plasma densities around $10^{20} \mathrm{~m}^{-}$

${ }^{3}$, and confinement times around $0.2 \mathrm{~ms}$. The plasma radius is about $0.23 \mathrm{~m}$ and the discharge duration up to a few ms. 
The Swarthmore Spheromak Experiment (SSX) at Swarthmore College, in operation since 1996, has performed scaling studies of the formation and equilibrium of spheromaks, showing in particular that spheromak formation is governed only by gun physics and is independent of the flux conserver dimension.

The SPHEX experiment (Rusbridge (1996)) was operated at the University of Manchester from 1989 to 1997 . The main issues considered were: the division of the plasma into a high electric field central column and a low electric field toroidal annulus; investigation of the global $n=1$ mode responsible for carrying energy and helicity from the central column to the annulus; the MHD dynamo responsible for current drive in the annulus. In the last years of activity the device was equipped with a central rod to improve stability. 


\subsection{Levitated dipole}

The last concept we would like to discuss is the magnetic dipole, a concept that has received so far limited attention but, on the basis of the present theoretical analyses, has a good potential to produce high- $\beta$ plasmas (Hasegawa 1987).

Space observations show that the equilibrium configuration consisting of a simple dipole field exhibit remarkable MHD stability properties, (with plasma beta exceeding unity e.g. in the Jupiter magnetosphere). Interchange modes can indeed be shown to be stable if the pressure profile decreases sufficiently slowly towards the low field region. Furthermore, if the equilibrium density and temperature have sufficiently weak gradients, as required by MHD stability, these free energy sources are not strong enough to drive small-scale instability and turbulent transport may be expected to be benign. In particular, the diamagnetic frequency tends to be smaller than the magnetic drift frequency resulting in a strong stabilizing effect (see e.g. Kesner 1998).

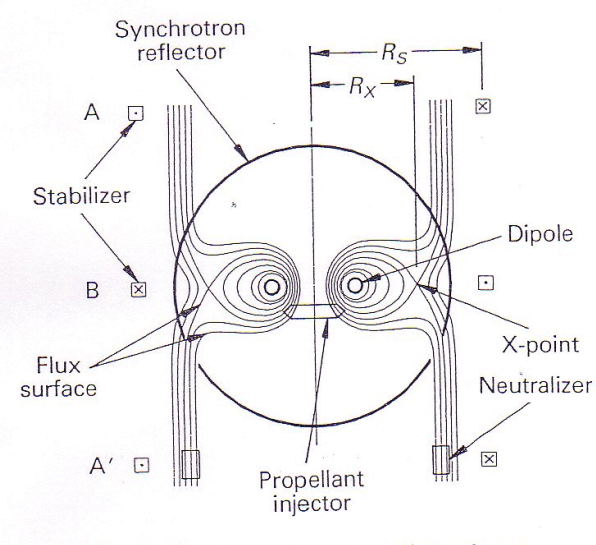

Fig. 2. Dipole reactor propulsion scheme.

Fig. 3.17 Levitated dipole (from Teller 1992)

A dipole configuration is produced by a large central coil which is levitated against gravity or local acceleration by a set of other coils, shown in Fig.3.17, that produce a vertical field. The combined field of the various coils produces a magnetic separatrix. Outside the separatrix a natural divertor configuration is formed. The presence of a magnetic separatrix can affect stability by enhancing MHD stability close to the separatrix and by locally destabilizing drift waves, although the latter could be stabilized by edge sheared flows similar to those observed in tokamaks in conjunction with improved confinement regimes.

Very little is known on the experimental side about dipole configurations. The levitated Dipole eXperiment (LDX), a facility with a superconducting ring of $0.4 \mathrm{~m}$ radius, is being constructed at MIT (Kesner 1998) with the aim of exploring plasmas with $300 \mathrm{eV}$ temperature and up to $10^{18} \mathrm{~m}^{-3}$ density. LDX started the operation at the end of 2004 .

The use of an internal coil surrounded by the plasma is the major drawback of the dipole configuration since no external cooling (or power supply) can be applied. Following an 
early suggestion by Dawson, the assumption usually made is that radiative cooling from the ring surface balances the heat input to the ring (coming from radiation, heat transport and neutrons) (the power needed for refrigerating the superconducting ring is also taken from the heat input through different energy conversion schemes). Note that since the surface heat temperature is limited by the material employed (e.g. $2700 \mathrm{~K}$ if the surface is made by tungsten), the above assumption sets a limit on the power that can reach the ring surface and therefore on the fusion power per unit volume.

The application for space propulsion has been considered in Teller (1992). The levitated dipole has a major radius of $6 \mathrm{~m}$ and a minor radius of $2 \mathrm{~m}$. The magnetic field on the conductor is $15 \mathrm{~T}$. The total fusion power $\left(\mathrm{D}^{3} \mathrm{He}\right.$ is considered) is $2 \mathrm{GW}, 60 \%$ of which available for thrust. With a total ring mass of 1180t, the resulting specific power is close to $1 \mathrm{~kW} / \mathrm{kg}$. Such a number, although of interest for space application, tends to fall on the low side. It is clear that improvements can arise from a minimization of the coil mass and on the use of materials allowing higher surface temperature and radiated power.

The design of the superconducting coil consists in a surface layer ( $1 \mathrm{~mm}$ thick) of tungsten, capable of radiating $1 \mathrm{MW} / \mathrm{m}^{2}$ at $2700 \mathrm{~K}$ for a total radiated power of $400 \mathrm{MW}$, followed by a shield of $\mathrm{C}-\mathrm{C}$ fiber composite (about $30 \%$ of the total ring mass) which attenuates $90 \%$ of the neutron flux (the total neutron power is about $60 \mathrm{MW}$ ), This firs shield is thermally insulated from a second shield structure made of two layers of $\mathrm{B}-\mathrm{H}_{2} \mathrm{O}$ steel structure (with a radial width/working temperature of $0.24 \mathrm{~m} / 900 \mathrm{~K}$ and $0.66 \mathrm{~m} / 300 \mathrm{~K}$, respectively) that attenuate the neutron flux by a factor 5600 , with only $467 \mathrm{~W}$ reaching the superconducting magnet working at $4.2 \mathrm{~K}$. Pumping all these powers from their working temperature up to the surface temperature of $2700 \mathrm{~K}$ requires, in ideal conditions, about $10 \mathrm{MW}$ of electric power that must be generated by converting the $400 \mathrm{MW}$ of input power to the ring. It should be stressed that the present design is only conceptual and that a deeper assessment is required to confirm these indicative figures. 


\subsection{Conclusions}

The mirror configuration may have some potential for application as a fusion propulsion system. Its geometry allows indeed to convert $50 \%$ of the fusion power in direct thrust power and the reminder in electricity by direct conversion, if advanced fuels are employed. Values of $\beta$ of interest for the use of advanced fuels can be achieved. Two main concepts are of interest and investigated. In this context:

- The feasibility of the tandem mirror concept has been experimentally proven in various devices. However, it requires sophisticated techniques to tailor the plugging potential and has been limited so far to low-density operation.

- The gasdynamic mirror concept is intrinsically simpler than the tandem mirror but requires very long systems or very high density values that may increase the neutron wall load beyond values today considered achievable within a mediumterm material development program for fusion application. A specific design for a propulsion system (possibly based on the ongoing design effort for a volumetric neutron source) could be undertaken to assess the potential of this concept on the basis of a more realistic set of assumptions on the relevant technologies.

Field Reversed Configurations can also produce direct thrust and electrical power directly. Their main feature is the possibility of achieving very high $\beta$ values, above $50 \%$. However, the stability of the configuration (observed so far only at large $\rho_{*}$ values) has still to be demonstrated at normalized Larmor radius $\rho_{*}$ of interest for energy production. Due to the very early stage of this line of research it is difficult to make reliable predictions about the global confinement capabilities. However, these configurations might be used for non-thermal fusion production schemes such as the recently proposed colliding beam fusion reactor. Such a configuration might simultaneously solve the formation/sustainment problem and take benefit from the presence of a population of fast ions to maintain the good stability properties of FRCs.

Conclusions on Magnetized Target Fusion cannot be made at this stage but the potential of this concept could be better assessed in a few years from now, when the results of the first experiments on FRX-L will be available.

Spheromaks have a geometry which also allows direct thrust and direct energy conversion. The capability of achieving high beta values is not as good as field reversed configurations but it might be adequate for space propulsion provided beta larger than $10 \%$ can be projected for reactor conditions. Plasma stability might require the insertion of a central conductor and the evolution of the configuration towards a ultra low aspect ratio tokamak (a configuration that requires a specific assessment). The main open issue is the capability of maintaining an effective dynamo mechanism with a minimum level of turbulence (laminar dynamo) to keep the energy confinement at an acceptable level. Encouraging results in this direction have been obtained in reversed field pinch experiments.

The Dipole configuration has very attractive features from the point of view of direct thrust/direct energy generation and achievable beta values. To assess its potential requires 
experimental results (which should come in the next few years from the LDX facility) on small scale stability and transport, although theoretical considerations indicate that a dipole could have good stability properties also at small scale. The most challenging technical issue is the construction of a superconducting coil capable of radiating all the incoming power and to produce the required electrical power needed for the coil refrigeration without excessive penalties on the coil mass.

In concluding this section we want to stress the possible interest of the spherical tokamak for space propulsion. This configuration (not considered in the present report since it is a closed magnetic field configuration) has the (already proven) advantage (in terms of confinement and stability) of the conventional tokamak but can achieve very high values of beta. The possibility of extracting particles for direct thrust has in principle the same difficulty of conventional tokamaks, where magnetic field lines do not escape from the reaction chamber and non trivial solutions must be investigated for the so called divertor. Note however that this possibility has been already considered for space propulsion and further studies might be beneficial in clarifying its potential. 


\section{Proposal for further studies on fusion for space application}

\subsection{Technology}

A number of assumptions made in this study are based on zero-order evaluation awaiting further refinement as suggested below.

Low-mass breeding blanket

The blanket (together with the magnet) can be a heavy component of the reactor core. Investigations performed for the SOAR conceptual design have pointed out that the minimum mass is achieved by using $\mathrm{LiH}$. On the basis of the experience gained in the last ten years on the design and $\mathrm{R} \& \mathrm{D}$ of blankets for fusion reactor applications, a detailed neutronic and thermal analysis should be made to assess the potential of this solution.

Low-mass magnet.

The magnet (together with the blanket) can be the heaviest component of the reactor core. Detailed design exists for magnets to be used in tokamak reactors, although these designs have not considered the constraints arising from the low mass requirements needed for space propulsion applications. A detailed design of a magnet for open magnetic field configurations should be made to benchmark the figures given in the generic fusion rocket studies both for superconducting and actively cooled copper magnets. The possible use of High Temperature Superconductors should be considered.

Auxiliary heating systems and cryoplant

All the fusion concepts rely on the use of auxiliary systems for heating the plasmas and of cryoplants for the superconducting magnets. The assumption made for the sake of illustration in generic fusion rocket studies $(1000 \mathrm{~kg}$ per $\mathrm{kW}$ of heat extracted for the cryoplant and $2.5 \mathrm{~kg}$ per $\mathrm{kW}$ of auxiliary power) need specific assessments. A substantial amount of $R \& D$ has been carried out in the fusion program on the main heating methods (neutral beam injection, ion cyclotron resonance heating, electron cyclotron resonant heating). The capability of low mass systems should be investigated together with high efficiency for power generation.

Radiator

Typical figures for the radiator specific power used in propulsion studies are in the range of $5 \mathrm{~kW}$ of radiated power for each $\mathrm{kg}$ of radiator mass. Since the radiator can be the heaviest component of the system, it is necessary to minimize its mass. Values in the range up to $100 \mathrm{~kW}$ of radiated power for each $\mathrm{kg}$ of mass can be envisaged. Radiator efficiency (e.g., power radiated/unit mass) depends on cycle temperature and material. To improve radiating power the temperature should be the highest compatible with cycle efficiency and material structural limits. At this time industrial practice for space power generation assumes "low" cycle temperatures of order 800-900 K. If sufficiently large power is available, there is no reason why the "low" temperature could be raised, using current high temperature ceramics (nitrides and carbides), to $1200^{\circ} \mathrm{C}$ without structural problems, reducing substantially radiator mass. This strategy has not yet been adopted or even tested, since known experience with large space power generators (say, > 
$20 \mathrm{~kW}$ ) is essentially nil. Nuclear space power generation will in fact have a deep impact on radiator technology. In any event, it seems advisable to investigate how to better exploit rejected heat prior to its disposal via a radiator, for instance utilizing thermionics or other more advanced physics. An assessment of the available technology should be made.

Thermal converter

Although the converter is typically not the heaviest component of the system, there is a wide range of estimate for the weight of the system.

Direct converter

A review of the present status of direct converters could provide better estimates of the achievable efficiencies and mass budgets.

Vacuum vessel/First wall

In present experiment the vacuum vessel is a not negligible part of the system mass. Applications to space propulsion require to minimize the mass and possibly to let part of the waste power to leave the configuration to avoid massive radiator systems. The aim of this study is to investigate the possibility that an electrically conducting wall (made by Mo, graphite or advanced carbon fiber) be transparent to 50-70\% of Bremsstrahlung and neutrons.

\section{Magnetic nozzle}

The conversion of high-energy charged particles into thrust depends on the design of the magnetic nozzle. The aim of this study is to critically review the presently proposed schemes in order to identify reasonable values for the conversion efficiency and point out possible problems in the magnetic nozzle design.

\subsection{Specific design studies}

\section{Colliding Beam Fusion Reactor}

The use of FRCs as a background neutralizer for non-thermal schemes that produce fusion power by beam-beam reactions (CBFR) has been proposed also for fusion propulsion (Cheung 2004). The aim of the proposed study is to perform a parameter optimization for space propulsion application and to critically review the assumption on the plasma dynamics introducing more general assumptions on the background plasma dynamics.

\section{Spherical Tokamak}

The spherical tokamak is a closed configuration and the extraction (for direct thrust) of high energy particles from the reaction chamber and the toroidal magnet is not trivial, although probably less difficult than in conventional tokamaks equipped with heavy magnets producing the toroidal magnetic field. Nevertheless already the existing medium scale experiments have shown the good potential of spherical tokamaks for energy production. Specific design studies (see Williams 1998) exist for space propulsion systems based on spherical tokamaks although the issue of particle extraction is not 
addressed in detail. The aim of the study should be to design a divertor configuration able to extract particles from the reaction chamber possibly looking at very low aspect ratio $(\mathrm{R} / \mathrm{a} \leq 1.5)$ equilibria.

Levitated dipole coils

As discussed in the previous section the levitated dipole coil must comply with the following requirements: high surface radiation (e.g. by high surface temperature), good neutron shielding of the superconducting magnet, efficient energy conversion of the incoming heat into electricity for the system refrigeration and low total mass. The present design is only conceptual and a further assessment could set a limit on the coil mass (and therefore on the foreseeable specific power). 


\section{Appendix A MISSIONS AND ESTIMATED PERFORMANCE}

Questions concerning the balance between $\mathrm{I}_{\mathrm{sp}}$ and power when planning interplanetary missions using fusion rockets can be better appreciated by looking quantitatively at their effect on propellant mass and $\Delta \mathrm{V}$. Note that these questions are not relevant to chemical propulsion, because thrust (applied for a very short time) is the variable controlling acceleration, not power. These are instead 'the' issues in fusion propulsion, where thrust may have to last for months or even years. In the following the fundamental assumption is that inert propellant is added to the fusion products, the purpose being to enhance thrust. Thrust due to fusion products only is, in fact, rather small, even though the Isp may be very large.

With this assumption, the relevant (and much simplified) equations to quantify fast missions made possible by fusion propulsion may be reduced to the following:

$$
\begin{array}{ll}
\mathrm{dm} / \mathrm{dt} & =\mathrm{F} / \mathrm{I}_{\mathrm{sp}} \\
\mathrm{M}_{\mathrm{p}} & =\mathrm{Ft}_{\mathrm{acc}} / \mathrm{I}_{\mathrm{sp}} \\
\mathrm{d}_{\mathrm{acc}} & =0.5 \mathrm{a}\left(\mathrm{t}_{\mathrm{acc}}\right)^{2} \\
\Delta \mathrm{V} & =\mathrm{a} \mathrm{t}_{\mathrm{acc}} \\
\mathrm{F} & =\mathrm{Ma}
\end{array}
$$

$\mathrm{I}_{\mathrm{sp}}$ definition

mass of propellant consumed at constant $\mathrm{dm} / \mathrm{dt}$ after a time tacc distance traveled at constant acceleration a $\Delta \mathrm{V}$ acquired after time $\mathrm{t}_{\text {acc }}$ at acceleration a Newton's law; $M$ is the total mass of the spacecraft

Then solving for time, mass $\mathrm{m}$ (that should be $<<\mathrm{M}$ in this example) and $\Delta \mathrm{V}$, we have:

$$
\begin{array}{ll}
\mathrm{t}_{\mathrm{acc}} & =\left(2 \mathrm{~d}_{\mathrm{acc}} \mathrm{I}_{\mathrm{sp}} \mathrm{M} / \mathrm{P}\right)^{1 / 2} \\
\mathrm{Mp} & =\left(2 \mathrm{~d}_{\mathrm{acc}} \mathrm{P} \mathrm{M} / \mathrm{I}_{\mathrm{sp}}^{3}\right)^{1 / 2} \\
\Delta \mathrm{V} & =\left(2 \mathrm{~d}_{\mathrm{acc}} \mathrm{P} /\left(\mathrm{MI}_{\mathrm{sp}}\right)\right)^{1 / 2}
\end{array}
$$

where $\mathrm{M}, \mathrm{d}_{\mathrm{acc}}$ and power $\mathrm{P}$ have been considered input parameters. For instance, the distance $\mathrm{d}_{\mathrm{acc}}$ may be half the distance, $\mathrm{d}$, to the destination chosen: in other words, the trajectory could consist of two portions: the first travelled at positive acceleration, a, until mid-way is reached, followed by deceleration until the spacecraft has reached a velocity sufficiently small to achieve orbit capture around the final destination. Alternatively, $\mathrm{d}_{\mathrm{acc}}$ is simply the distance travelled at positive acceleration, after which the spacecraft may coast at constant speed (it is understood then that at some convenient point the inverse strategy must take place to slow down the spacecraft, for instance, with the same deceleration, $-\mathrm{a}$, of the first example). However, $\mathrm{d}_{\mathrm{acc}}$ may turn out to be much greater than $\mathrm{d}$ when the acceleration $\mathrm{a}=\mathrm{P} /$ (Isp $\mathrm{M}$ ) is very small, i.e., at very low thrust and power. In this case the spacecraft must spiral (for instance, around Earth, or around the planet or star reached, starting the return leg of the trip), until reaching the right escape $\Delta \mathrm{V}$. Only then the ship can start accelerating along the trans-planetary trajectory.

In the solution set above note the favorable effect of Isp on propellant mass and its opposite effect on acceleration time and $\Delta \mathrm{V}$ : in fact, at fixed power, increasing thrust comes at the expense of decreasing Isp, so it takes longer and longer to reach smaller and smaller $\Delta \mathrm{V}$. 
To show the potential, and limitations, posed by powered trajectories, consider propulsion solutions for a nominal Earth to Mars mission (minimum Earth to Mars distance, $\mathrm{d}$, is about $1.5 \times 10^{8} \mathrm{~km}$; for the purpose of illustration, the distance $\mathrm{d}$ chosen is doubled to $3 \times 10^{11} \mathrm{~m}$, and the trip is assumed to consist of two equal portions, as in the first example mentioned.

The matrix of input data is: $\mathrm{M}=10^{2}$ and $10^{3} \mathrm{t}$; Isp $=10^{5}, 10^{6}$ and $10^{7} \mathrm{~m} / \mathrm{s}$, and thrust power $\mathrm{P}=1,10$ and $100 \mathrm{GW}$. The results are in Figures A1 and A2, plotting on log-log scales the propellant mass $\mathrm{m}$, the acceleration time (tacc) and the _ $\mathrm{V}(\mathrm{Dv})$ as a function of $I_{s p}($ in $\mathrm{m} / \mathrm{s}$ ) for the two spacecraft masses $\mathrm{M}=100$ and $\mathrm{M}=1000 \mathrm{t}$.

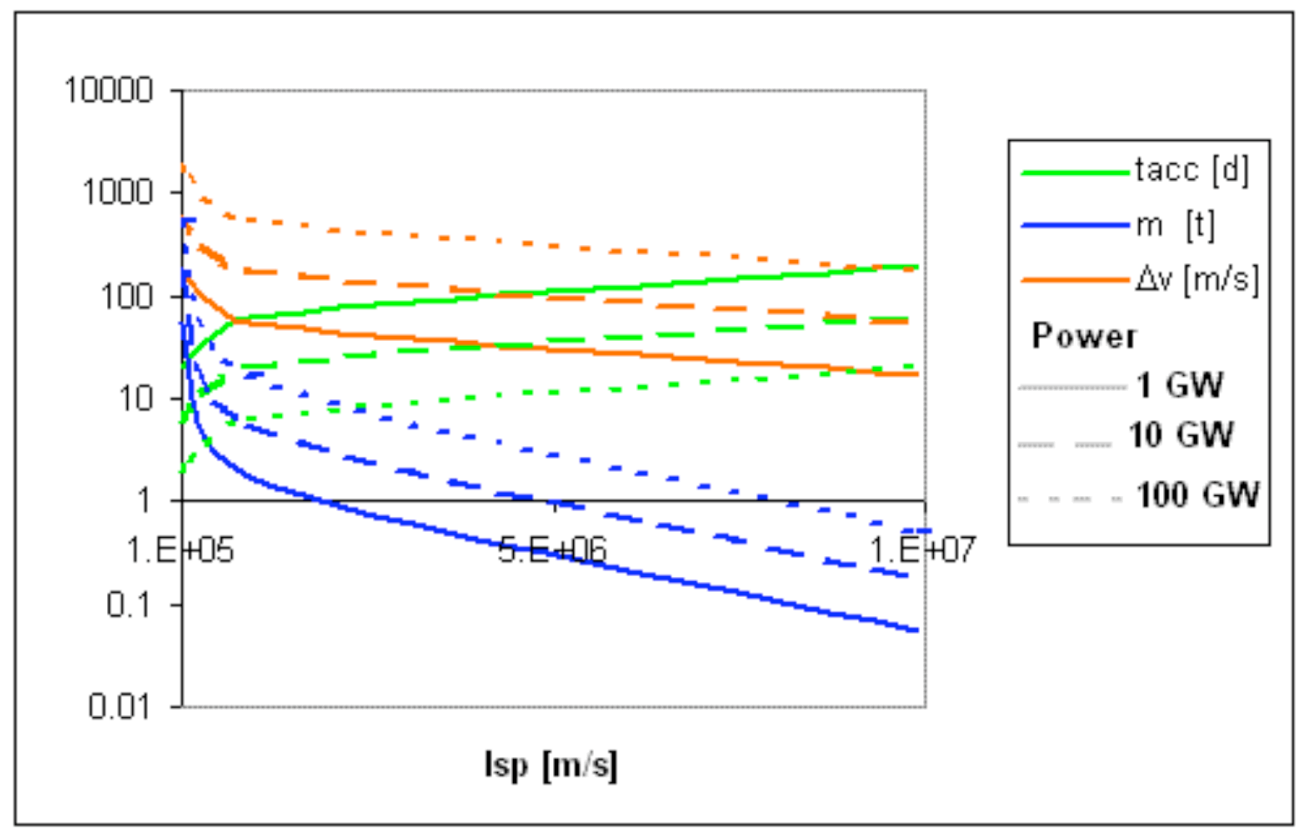

Fig.A1 Spacecraft velocità increment, acceleration time and propellant consumed as a function of $\mathrm{I}_{\mathrm{sp}}$ for a $100 \mathrm{t}$ spacecraft 


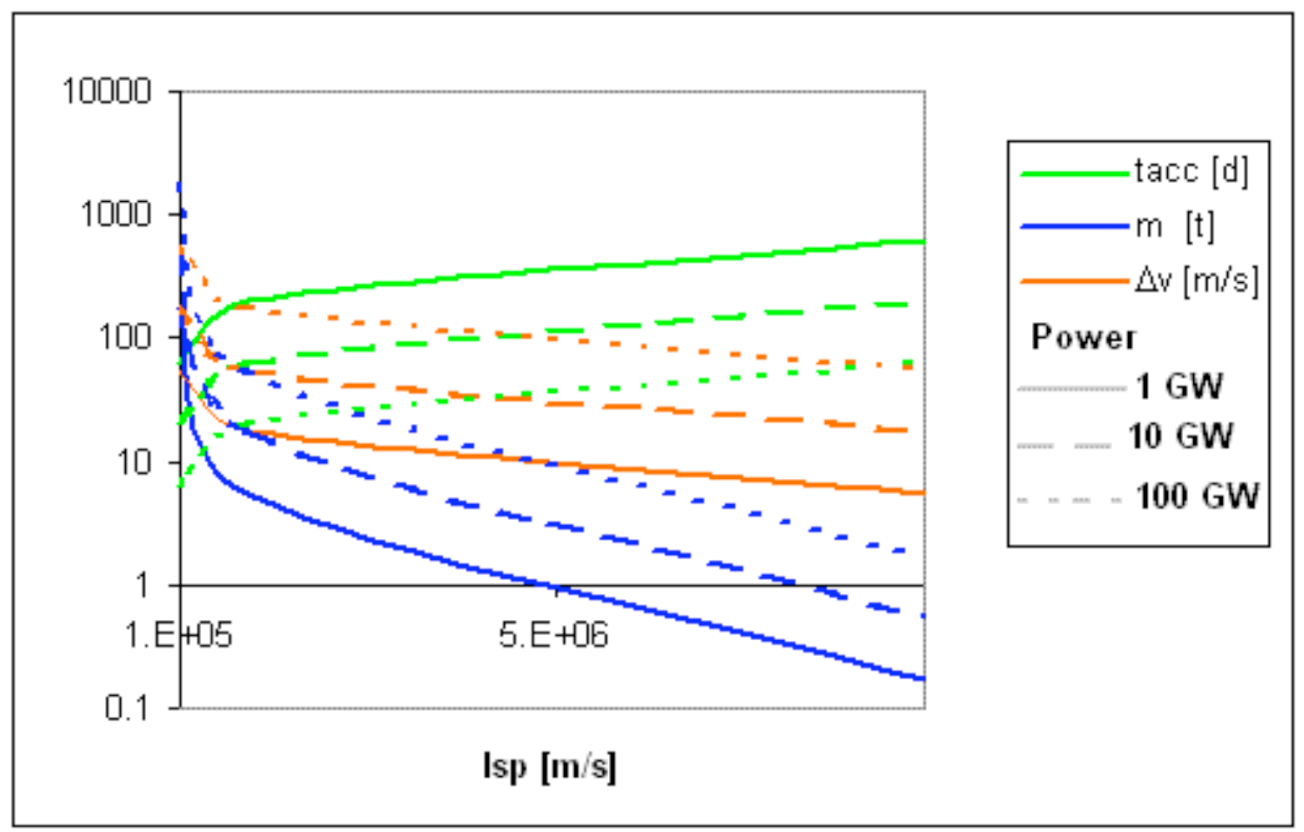

Fig. A2 Spacecraft velocità increment, acceleration time and propellant consumed as a function of $I_{\text {sp }}$ for a $1000 \mathrm{t}$ spacecraft

The curves indicate the sharp increase in consumed propellant near the lowest $I_{\mathrm{sp}}$. However, with a modest $I_{\mathrm{sp}}=10^{5} \mathrm{~m} / \mathrm{s}$ and for the higher spacecraft mass, the mission is doable and practical with $\mathrm{P}=1 \mathrm{GW}$. The $\mathrm{M}=100 \mathrm{t}$ case is not doable under the assumptions made ( $\mathrm{m}$ is of the same order of $\mathrm{M}$ ).

At the intermediate $\mathrm{I}_{\mathrm{sp}}=10^{6} \mathrm{~m} / \mathrm{s}$, both spacecraft can perform a mission in reasonable times, the best being the case of $M=100 \mathrm{t}$ and $\mathrm{P}=10 \mathrm{GW}$. The highest $\mathrm{I}_{\mathrm{sp}}\left(10^{7} \mathrm{~m} / \mathrm{s}\right)$ poses quite a propulsion challenge; once met and successfully overcome, it enables fast missions only at the highest power, that is, $100 \mathrm{GW}$. Scaling of open magnetic fusion reactors/thrusters is not established with the same level of confidence of tokamaks, so at the highest power there are questions concerning how to design, build and operate such reactors.

From this crude example it seems that a reasonable preliminary design of a fusion rocket for a fast Mars mission involves thrust power of order $10 \mathrm{GW}$ with $\mathrm{I}_{\mathrm{sp}}$ of order $10^{6} \mathrm{~m} / \mathrm{s}$ and a spacecraft of order 100 ton. Since the trip would last no more than 20 days, this mass may be adequate, if the reactor may be made sufficiently compact and light. If that is not feasible, and mass must be of order $10^{3} \mathrm{t}$, a practical 'fast' Mars mission is possible only with a modest $\mathrm{I}_{\mathrm{sp}}=10^{5} \mathrm{~m} / \mathrm{s}$ rocket using $1 \mathrm{GW}$ power. 


\section{References}

Bodin, H.A.B. and Newton, A. A., (1980), "Reversed Field Pinch research" Nucl. Fusion 201255

Borowski, S.K., (1995), "Comparison of Fusion/Antiproton Propulsion Systems for Interplanetary Travel”, in: Fusion Energy for Space Propulsion, ed. by T. Kammash, AIAA Progress in Astronautics and Aeronautics Series, Vol. 167, Washington, Ch. 4.

Bussard, R.W., (1990), "Fusion as Electric Propulsion", J. of Propulsion, Vol. 6, No. 5, pp. 567-574.

Carpenter, S.A. and Deveny, M.E., (1992) 'Mirror Fusion Propulsion System (MFPS): An Option for the Space Exploration Initiative (SEI)," 43rd Congress of the Int. Astronautical Federation, paper IAF-92-0613 (Washington, DC, 28 August--5 September, 1992).

Carpenter, S. and Deveny, M., (1993) "Overview of the mirror fusion propulsion system (MFPS): an optimised open magnetic field configuration using D3He", Proc. Second Wisconsin Symposium on Helium-3 and fusion power, WCSAR-TR-AR3-9307-3

Casali, D., and Bruno, C., (2004), "Superconducting Materials Applied to Electric propulsion", AIAA J. Spacecrafts and Rockets, Vol. 41, No. 4, July-August 2004.

Chapman, M., Miley, G.H., and Kernbichler, W., (1989) 'Fusion Space Propulsion with a Field Reversed Configuration," Fusion Technology 15, 1154.

Cheung, A. at al. (2004) "Colliding beam fusion reactor space propulsion system" STAIF-2004, Volume 699, pp.354-361

Cho, T., Higaki, H., Hirata, M., Hojo, H., Ichimura, M., Ishii, K., et al. (2004) “Advances in Potential Formation and Findings in Sheared Radial Electric-Field Effects on Turbulence and Loss Suppression in GAMMA 10" Proc. $20^{\text {th }}$ IAEA Fusion Energy Conference, Vilamoura 2004, paper EX/9-6Rd

Cox, L.T., et al. (1990) "Thermonuclear reaction listing with cross-section data for four advanced reactions", Fusion Technol. 18, 325

Emrich, W.J. (2002), "Current status of the gas-dynamic mirror fusion propulsion experiment" SPACE TECHNOLOGY AND APPLICATIONS INTERNATIONAL FORUM-STAIF 2002. Albuquerque, NM, 3-6 February, 2002. Edited by Mohamed S. ElGenk. Melville, NY: American Institute of Physics, 2002. AIP Conference Proceedings, Volume 608, pp. 801-806 (2002).

Fusion Energy Science Advisory Committee (FESAC) (2003) "Non electric application of fusion" 
Gormezano, C. (1979) "Reduction of losses in open-ended magnetic traps" Nucl. Fusion 19, 1085

Hasegawa, A. (1987), “A Dipole Field Fusion Reactor”, Comm. On Plasma Phys and Controlled Fusion, 1, 147

Hoffman, A.L., Guo, H.Y., Miller, K.E., Milroy, R.D., (2004) "Long Pulse FRC Sustainment with Enhanced Edge Driven Rotating Magnetic Field Current Drive", Proc. $20^{\text {th }}$ Fusion Energy Conference, Vilamoura 2004, paper IC/P6-41

Huguet, M. (2002) "The ITER magnets: preparation for full size construction based on the results of the model coil programme" Proc. $19^{\text {th }}$ Fusion Energy Conference, Lyon 2002, IAEA

Jarboe, T.R., (1994) "Review of Spheromak research", Plasma Physics and Controlled Fusion, 36, p. 945

Kadomtsev B. B. (1975), “Tokamaks and dimensional analysis” Sov. J. Plasma Physics, 1, 295

Kammash, T., (1995a), "Principles of Fusion Energy Utilization in Space propulsion", in: Fusion Energy for Space Propulsion, ed. by T. Kammash, AIAA Progress in Astronautics and Aeronautics Series, Vol. 167, Washington, Ch. 1.

Kammash, T., et al., (1995b)"High-Performance Fusion Rocket for Manned Space Missions", in: Fusion Energy for Space Propulsion, ed. by T. Kammash, AIAA Progress in Astronautics and Aeronautics Series, Vol. 167, Washington, Ch. 1.

Kaufmann, III, W.J., (1993), "Discovering the Universe", 3rd ed., W.H. Freeman and Co., NY, Ch. 17 and Appendix 4.

Kawamori, E., Murata, Y., Umeda, K., Hirota, D., et al., (2004) "Ion Kinetic Effect on Bifurcated Relaxation to a Field-Reversed Configuration in TS-4 CT experiment", Proc. 20 ${ }^{\text {th }}$ Fusion Energy Conference, Vilamoura 2004, paper EX/P5-7

Kesner, J., Bromberg, L., Garnier, D., Mauel, M., (1998) "Plasma Confinement in a magnetic dipole", Proc. $17^{\text {th }}$ Fusion Energy Conference, Yokohama 1998, paper ICP-09

Kruglyakov, E.P., Dimov, G.I., Ivanov, A.A., Koidan V.S., (2002) “Axisymmetric Magnetic Mirrors for Plasma Confinement. Recent Development and Perspectives", Proc. $19^{\text {th }}$ Fusion Energy Conference, Lyon 2002, paper EX/C1-4Rb

Kulcinski, G.L., (1987) et al. "SOAR Space Orbiting Advanced Power Reactor" UWFDM-722 
Kulcinski, G.L., Ashley, R.P., Santarius, J.F., Piefer, G., Subramanian, K.M., (2000) "The development of lunar 3He resources: near term applications and long term prospects", UWFDM-1128

Miley, G.H., et al. (1978) "Preliminary design of a self-sustained advanced-fueld field reversed mirror reactor-SAFFIRE" Trans. Am. Nucl. Soc. 30, 47

Miley, G.H., (1987) Advanced propulsion power- a preliminary assessment Air Force study board, NAS, NAS Press, Washington D.C.

Mirnov, V.V. and Ryutov, D.D. (1979) "Linear gasdynamic system for plasma confinement" Sov. Tech. Phys. Lett. 5, 279

Nagornyj, V.P., Ryutov, D.D., and Stupakov, G.V. (1984), "Flute instability of plasma in a gas dynamic trap", Nucl. Fusion, 24, 1421

Nakashima, H., Miley, G.H., and Nakao, Y., (1994) 'Field Reversed Configuration (FRC) Fusion Rocket," Proc. 11th Symp. Space Nuclear Power and Space Propulsion Systems (Albuquerque, NM, 1994).

Okada, S., Fukuda, T., Kitano, K., Sumikura, H., et al. (2004) "Sustainment and Additional Heating of High Beta Field Reversed Configuration Plasmas", Proc. 20 Fusion Energy Conference, Vilamoura 2004, paper IC/P6-6

Ozima, M., Seki, K., Terada, N., Miura, Y.N., Podosek, F.A., and Shinagawa, H., (2005), "Terrestrial nitrogen and noble gases in lunar soils", Nature, Vol. 436/4, pp. 655-659, 4 August 2005.

Post, R.F., (1987) “The magnetic mirror approach to fusion”, Nuclear Fusion, 27, pp.1579-1739

Rechester, A.B. and Rosenbluth, M.N. , (1978) Phys. Rev. Lett 4038

Rostoker, N., et al. (1993) "Magnetic fusion with high energy self colliding ion beams" Phys. Rev. Lett. 70, 1818.

Rostoker, N., Qerushi, A., Binderbauer, M., (2003) "Colliding beam fusion reactors", Journal of Fusion Energy, 83

Roth, J.R., (1989) “'Space Applications of Fusion Energy," Fusion Technology 15, 1375.

Rusbridge, M. G., Gee, S. J., Browning, P. K., Cunningham, G., et al. (1996) "The design and operation of the SPHEX spheromak", Plasma Physics and Controlled Fusion, 39, 683 
Santarius, J.F. et al. (1988), "Critical Issues for SOAR: The Space Orbiting Advanced Fusion Reactor," in Space Nuclear Power Systems 1988, M. S. El-Genk and M. D. Hoover, Eds. Malabar, FL.: OrbitBook Company, 1989, pp. 167.

Santarius, J.F. and Logan, B.G., (1998) "Generic magnetic fusion rocket", Journal of propulsion and power 14, 519

Sarff, J.S., Almagri, A.F., Anderson, J.K., Biewer, T.M., et al., (2002) "Overview of improved confinement and plasma control in the MST reversed field pinch", Proc. 19 Fusion Energy Conference, Lyon 2002, paper OV/4-3

Schulze, N.R. and J.R. Roth, (1991) "The NASA-Lewis Program on Fusion Energy for Space Power and Propulsion, 1958-1978," Fusion Technology 19, 11.

Schulze, N.R. (1994) "Figures of Merit and Attributes for Space Fusion Propulsion," Fusion Technology 25, 182.

Siemon, R., Peterson, P., Ryutov, D., Kirkpatrick, R., et al. “The relevance of Magnetized Target Fusion (MTF) to practical energy production" LA-UR-99-2956

Simonen, T.C., et al. "TMX-U Thermal-Barrier Experiments", IEEE Trans. Plasma Science 16, 1

Steinhauer, L.C. et al., (1998) “White paper on FRC development” April 1998

Stuhlinger, E. (1964) “Ion propulsion for space flight” McGraw-Hill, New York

Taccetti, J. M., Intrator, T. P., Wurden, G. A., Zhang, S. Y., et al (2003) "FRX-L: A Field-Reversed Configuration Plasma Injector for Magnetized Target Fusion", Rev. Sci. Instrum., 74, 4314

Taylor, J.B., (1976) "Relaxation of toroidal discharges", in Pulsed high beta plasmas Ed. D.E. Evans, Pergamon, New York, 59

Teller, E., Glass, A.J., Fowler, T.K., Hasegawa, A., and Santarius, J.F. (1992) 'Space Propulsion by Fusion in a Magnetic Dipole," Fusion Technology 22, 82.

Thio, Y. C. F., Freeze, B., Kirkpatrick, R. C., Landrum, B., Gerrish, H. and Schmidt, G. R. (1999) "High-Energy Space Propulsion based on Magnetized Target Fusion.," 35th AIAA Joint Propulsion Conference and Exhibit, June 20-24, 1999, Los Angeles, CA. Paper AIAA-99-2703.

Tuszewski, M. (1988) “Field reversed configurations” Nucl. Fusion 28, 2033.

Williams, C.H. et al. (1998) “A spherical torus nuclear fusion reactor space propulsion vehicle concept for fast interplanetary travel” AIAA-98-3591 
Wood, R.D.. Cohen, B.I., Hill, D.N., Cohen, R.H.. et al. (2004) "Improved operation and modeling of the SSPX spheromak", Proc. $20^{\text {th }}$ Fusion Energy Conference, Vilamoura 2004, IAEA, paper EX/9-5 